\title{
Viscoacoustic anisotropic wave equations
}

\author{
Qi Hao* and Tariq Alkhalifah ${ }^{\dagger}$ \\ * King Fahd University of Petroleum and Minerals (KFUPM) \\ College of Petroleum Engineering and Geosciences \\ Dhahran, Saudi Arabia \\ E-mail:xqi.hao@gmail.com,qi.hao@kfupm.edu.sa \\ $\dagger$ King Abudulah University of Science and Technology (KAUST) \\ Division of Physics and Engineering \\ Thuwal, Saudi Arabia \\ E-mail: tariq.alkhalifah@kaust.edu.sa \\ (February 13, 2020) \\ Running head: Viscoacoustic anisotropic wave equations
}

\begin{abstract}
The wave equation plays a central role in seismic modeling, processing and inversion. Incorporating the attenuation anisotropy into the acoustic anisotropic wave equations provides a choice for acoustic modeling and imaging in fluid-filled anisotropic reservoirs. However, the existing viscoacoustic anisotropic wave equations are obtained for a specified viscoacoustic model. In this paper, we present a relatively general representation of the scalar and vector viscoacoustic wave equations for orthorhombic anisotropy. We also show the viscoacoustic wave equations for transverse isotropy as a special case. The viscoacoustic orthorhombic wave equations are flexible for multiple viscoacoustic models. We take into account the classic visocoacoustic models such as the Kelvin-Voigt, Maxwell, standard-linear-solid
\end{abstract}


and Kjartansson models, and derive the corresponding viscoacoustic wave equations in differential form. To analyze the wave propagation in viscoacoustic models, we derive the asymptotic acoustic point-source radiation. Numerical examples show a comparison of the acoustic waveforms excited by a point source in the viscoacoustic orthorhombic models and the corresponding nonattenuating model, and the effect of the attenuation anisotropy on the acoustic waveforms. 


\section{INTRODUCTION}

Linear viscoelasticity has been widely used in describing anelastic behaviors of rocks. For linear viscoelastic media, the time-domain constitutive equation describing the relation between stress and strain is expressed in a mathematical form by Boltzmann superposition principle (Lakes, 2009). The stress depends on the whole history of the strain, vice versus. The consitutive equation is characterized by relaxation or creep functions. The theory of linear viscoelasticity is discussed systematically in Hudson (1980), Christensen (1982), Pipkin (1986), Tschoegl (1989), Borcherdt (2009), Lakes (2009) and Carcione (2015).

For one-dimesional viscoelastic media, some elementary viscoelastic models can be built by a specified combination of an elastic spring and a viscous dash-pot. The stress-strain relation for the spring is purely elastic, while the one for the dash-pot is viscous describing the behavior of a Newtonian fluid. The Kelvin-Voigt model is described by connecting a spring and a dash-pot in parallel. The Maxwell model is obtained by connecting a spring and a dash-pot in series. The standard-linear-solid (SLS) model, also called the Zener model, can be formed by connecting a spring in series with a Kelvin-voight model. All of these models have the behavior of frequency-dependent quality factor. By contrast, the Kjartansson model has a power-law relaxation function (Kjartansson, 1979) and the quality factor for the model is independent of frequency. Hence, this model is also called the constant Q model. As mentioned in Schiessel et al. (1995), the dynamical property of the Kjartansson model is intermediate between the features of the elastic solid (spring) and viscous liquid (dash-pot). The Kjartansson model can be used as a fractional element to construct the fractional Kelvin-Voigt, Maxwell and SLS models (Schiessel et al., 1995). Extending some of these models to the anisotropic case can be seen in Carcione (2015), Bai 
and Tsvankin (2016), Bai et al. (2017) Zhu (2017), Zhu and Bai (2019).

Studying wave propagation in a homogeneous viscoelastic medium can be realized by calculating the point-source radiation as the solution of the viscoelastic wave equation. According to the correspondence principle (Ben-Menahem and Singh, 1981), the exact solution of the viscoelastic wave equation for a time harmonic point source can be obtained by substituting the viscoelastic medium parameters into the solution of the corresponding elastic wave equation. For an elastic anisotropic medium, the exact point-source radiation can be found in Yeatts (1984), van der Hijden (1987), Burridge et al. (1993), and Wang and Archenbach (1995). The steepest-descent and stationary-phase methods are efficient tools to derive the asymptotic approximation for the point-source radiation (Buchwald, 1959; Lighthill, 1960; Vavryčuk, 1997, 2007a). The point-source radiation for a homogeneous viscoelastic anisotropic medium can be obtained by analogy with the derivation of the point-source solution for an elastic medium. Bleistein (2012) used the steepest-descent method to derive an approximate formula for the $n$-fold complex-valued integral. Shekar and Tsvankin (2014) implemented Bleistein (2012)'s formula to derive an asymptotic pointsource radiation for a viscoelastic anisotropic medium. Vavryčuk (2007a) demonstrated the validity of the asymptotic approximation for the Green function for a viscoelastic anisotropic medium.

The acoustic anisotropic wave equations (Alkhalifah, 2000, 2003) for nonattenuating TI (transversely isotropic) and orthorhombic media have been widely implemented in exploration seismology for anisotropic media. As an alternative to the scalar form of the acoustic anisotropic wave equations (Alkhalifah, 2000, 2003), a vector form of the acoustic wave equations can be found in Duveneck and Bakker (2011) for TI media and Fowler and King (2011) for orthorhombic media. Although both forms of the acoustic anisotropic wave 
equations are based on the acoustic approximation (i.e. the shear-wave velocity parameter $v_{S 0}$ equals to zero), the scalar acoustic anisotropic wave equations are obtained from the dispersion relation, and the vector acoustic anisotropic wave equations are derived from a combination of the equation of motion, the relation between stress and displacement, and the constitutive equation. Incorporating attenuation anisotropy (Zhu and Tsvankin, 2006, 2007) into the acoustic anisotropic eikonal equations prompts the development of the acoustic wave equations. Xu et al. (2016) and da Silva et al. (2019) obtained different vector forms of the viscoacoustic TI wave equation for the generalized SLS model. Qu et al. (2017) incorporated attenuation terms into the acoustic TI wave equation (Duveneck and Bakker, 2011), and derived a pseudo-differential viscoacoustic TI wave equation. All of these viscoacoustic wave equations naturally reply on a specified viscoacoustic model.

The aim of this paper is to show a relatively general representation of the viscoacoustic wave equations for orthorhombic anisotropy in scalar and vector forms. These viscoacoustic wave equations do not reply on a specified viscoacoustic model. In the frequency domain, the viscoacoustic wave equations have the same form as the acoustic wave equations for nonattenuating TI and orthorhombic media. In the time domain, the Riemann-Stieltjes integral (Apostol, 1974) is involved in the viscoacoustic wave equations. The approximate formula for the $n$-fold complex-valued integral (Bleistein, 2012) is implemented to derive the asymptotic P-wave point-source radiation for the scalar viscoacoustic wave equation.

In this paper, we will switch between the time-space and frequency-wavenumber domains. The Fourier transform of the time-space domain wavefield $f(\mathbf{x}, t)$ is written as

$$
\hat{f}(\mathbf{k}, \omega)=\int_{-\infty}^{\infty} \int_{-\infty}^{\infty} \int_{-\infty}^{\infty} \int_{-\infty}^{\infty} f(\mathbf{x}, t) e^{i\left(\omega t-k_{1} x-k_{2} y-k_{3} z\right)} \mathrm{d} x \mathrm{~d} y \mathrm{~d} z \mathrm{~d} t
$$

and the inverse Fourier transform of the frequency-wavenumber domain wavefield $\hat{f}(\mathbf{k}, \omega)$ 
is written as

$$
f(\mathbf{x}, t)=\frac{1}{(2 \pi)^{4}} \int_{-\infty}^{\infty} \int_{-\infty}^{\infty} \int_{-\infty}^{\infty} \int_{-\infty}^{\infty} \hat{f}(\mathbf{k}, \omega) e^{i\left(k_{1} x+k_{2} y+k_{3} z-\omega t\right)} \mathrm{d} k_{1} \mathrm{~d} k_{2} \mathrm{~d} k_{3} \mathrm{~d} \omega
$$

where $\mathbf{x}=(x, y, z)^{T}$ and $\mathbf{k}=\left(k_{1}, k_{2}, k_{3}\right)^{T}$ denote the position and wavenumber vectors, respectively, $t$ and $\omega$ denote the time and the angular frequency, respectively.

In addition, we will use the Riemann-Stieltjes integral (Apostol, 1974) to derive the viscoacoustic wave equations. Referring to Carcione (2015), we define the operation " $\odot$ " of two functions of the time as

$$
g(t) \odot s(t) \equiv g(t) * \mathrm{~d} s(t)=\int_{-\infty}^{t} g(t-\tau) \mathrm{d} s(\tau)
$$

to describe the Riemann-Stieltjes convolution integral. Here, $g(t)$ and $s(t)$ are functions of the time, $g(t)$ is assumed to be causal, and the operation "*" denotes the time convolution.

An important property of the operation is

$$
g(t) \odot s(t)=s(t) \odot g(t)
$$

If any one of these two functions is a constant, the operation $\odot$ becomes a product, that is

$$
\kappa \odot s(t)=s(t) \odot \kappa=\kappa s(t)
$$

where $\kappa$ is a constant.

More properties about the Riemann-Stieltjes convolution integral can be found in Gurtin and Sternberg (1962) and Apostol (1974).

\section{CONSTITUTIVE EQUATION}

For a viscoelastic medium, the stress at a point depends on the whole time history of the strain at the point. The relation between the stress and strain is causal, that is the current 
values of the stress do not depend on the future values of strain (Hudson, 1980). Assuming the medium to be homogeneous and anisotropic, the constitutive equation relating the stress and the strain is written as the Riemann-Stieltjes convolution integral

$$
\sigma_{i j}(\mathbf{x}, t)=\psi_{i j k l}(t) \odot e_{k l}(\mathbf{x}, t)
$$

where the operation $\odot$ is defined in equation $3, t$ denotes the time, $\mathbf{x}$ denotes the spatial position, $\sigma_{i j}$ denote the second-order stress components, $e_{k l}$ denote the second-order strain components, $\psi_{i j k l}$ denote the fourth-order relaxation functions, and the repeated indexes satisfy the Einstein summation convention. The relaxation functions describe the stress response to a unit strain applied at the time $t=0$. Hence, the relaxation functions are required to be a non-increasing causal function of the time.

We assume that the medium starts to oscillate at the time $t_{0}=0$ and no historical strain exists for $t \leq 0$ throughout the paper. In the case that the relaxation functions are bounded when the time approaches zero from the positive time axis, that is $\left|\psi_{i j k l}(0+)\right|<\infty$, the Riemann-Stieltjes convolution integral can be rewritten as (Gurtin and Sternberg, 1962; Hudson, 1980)

$$
\psi_{i j k l}(t) \odot e_{k l}(\mathbf{x}, t)=\psi_{i j k l}(0+) e_{k l}(\mathbf{x}, t)+\int_{0}^{t} \dot{\psi}_{i j k l}(t-\tau) e_{k l}(\mathbf{x}, \tau) \mathrm{d} \tau
$$

where " $0+$ " means approaching to zero from the positive time axis, $\psi_{i j k l}(0+)$ is the instantaneous elastic stiffness coefficients, and the dot on $\dot{\psi}_{i j k l}$ denotes the first derivative with respect to the time $t$. The above equation is applicable for the Kelvin-Voigt, Maxwell and SLS models shown in the section "Viscoacoustic orthorhombic models and the corresponding wave equations".

In the case that the relaxation functions are infinite when the time approaches zero from the positive time axis, that is $\left|\psi_{i j k l}(0+)\right|=\infty$, equation 7 cannot be used to compute the 
stress. We have to utilize equation 6 and deal with the singularity at $t=0$ for a specific relaxation function. This case often appears in the viscoelastic/viscoacoustic models with the relaxation functions of the power-law form, such as the Kjartansson model (see the density-normalized relaxation functions as illustrated in equation 39). For the Kjartansson model, the Riemann-Stieltjes convolution integral can be rewritten as

$$
\psi_{i j k l}(t) \odot e_{k l}(\mathbf{x}, t)=\int_{0}^{t} \dot{\psi}_{i j k l}(t-\tau) e_{k l}(\mathbf{x}, \tau) \mathrm{d} \tau
$$

Here, the contribution of the singular term $\psi_{i j k l}(0+) e_{k l}(\mathbf{x}, t)$ to the result is not taken into account, because the output of a physical system must be finite. This treatment of the singular term can be verified in Kjartansson (1979).

Applying Fourier transform to equation 6 and taking account of equations 7 and 8, we derive the frequency-domain constitutive relation between the stress and strain,

$$
\hat{\sigma}_{i j}(\mathbf{x}, \omega)=c_{i j k l}(\omega) \hat{e}_{k l}(\mathbf{x}, \omega)
$$

where $\omega$ denotes the angular frequency. The complex stiffness coefficients $c_{i j k l}$ are given by

$$
c_{i j k l}(\omega)= \begin{cases}\psi_{i j k l}(0)+\int_{0}^{\infty} \dot{\psi}_{i j k l}(t) e^{i \omega t} \mathrm{~d} t, & \text { if }\left|\psi_{i j k l}(0+)\right|<\infty \\ \int_{0}^{\infty} \dot{\psi}_{i j k l}(t) e^{i \omega t} \mathrm{~d} t, & \text { if }\left|\psi_{i j k l}(0+)\right|=\infty\end{cases}
$$

where the integral at the right side of the above equation describes the Fourier transform of the first temporal derivative of $\psi_{i j k l}$, and the case $\left|\psi_{i j k l}(0+)\right|=\infty$ corresponds to the Kjartansson model specifically.

\section{VISCOACOUSTIC WAVE EQUATIONS}

In this section, we shall describe the viscoacoustic orthorhombic wave equations in scalar and vector forms. We use the two-index notation $\phi_{i j}$ to denote the density-normalized relaxation functions in an orthorhombic medium. The density-normalized stiffness coefficients 
as functions of frequency are denoted by $a_{i j}(\omega)=a_{i j}^{R}(\omega)-i \operatorname{sgn}(\omega) a_{i j}^{I}(\omega)$, where the superscripts $R$ and $I$ denote the real and imaginary parts of the stiffness coefficients, respectively, and the minus sign "-" corresponds to the Fourier transform convention over $t$ (equation 1). The elements of the quality factor matrix are given by $Q_{i j}(\omega)=a_{i j}^{R}(\omega) / a_{i j}^{I}(\omega)$.

We first combine Tsvankin's (1997) and Zhu and Tsvankin's (2007) notations to describe the stiffness coefficients in the frequency domain, where the parameters in these two notations are dependent on frequency. We then utilize the acoustic approximation to set the shear-wave velocity parameter $v_{S 0}$ equal to zero for all frequencies. As a result, the stiffness coefficients associated with P-waves are independent of the shear-wave velocity $v_{S 0}$ and anisotropy parameters $\gamma_{1}$ and $\gamma_{2}$ in Tsvankin's (1997) notation, and of the shearwave attenuation parameter $A_{S 0}$ and attenuation-anisotropy parameters $\gamma_{Q 1}$ and $\gamma_{Q 2}$ in Zhu and Tsvankin's (2007) notation, where the subscripts "1" and "2" denote the $x$ - and $y$ axes normal to the $[y, z]$ and $[x, z]$ symmetry planes, respectively. We next use the modified Alkhalifah's (2003) notation (see equations A-1 to A-6 in Appendix A) to replace Tsvankin's (1997) notation. Finally, all of these operations give rise to the density-normalized stiffness coefficients in terms of the parameters in the modified Alkhalifah's (2003) and Zhu and Tsvankin's notations (2007) as follows,

$$
\begin{aligned}
& a_{11}(\omega)=v_{n 2}^{2}\left[1-2 i k_{Q}\left(1+\epsilon_{Q 2}\right)\right]\left(1+2 \eta_{2}\right) \\
& a_{12}(\omega)=v_{n 1} v_{n 2} \xi\left[1-2 i k_{Q}\left(1+\epsilon_{Q 2}\right)\right]-i k_{Q} \delta_{Q 3}\left(1+\epsilon_{Q 2}\right) \frac{v_{n 2}^{3}\left(1+2 \eta_{2}\right)^{2}}{v_{n 1} \xi} \\
& a_{13}(\omega)=v_{P 0} v_{n 2}\left(1-2 i k_{Q}\right)-i k_{Q} \delta_{Q 2} \frac{v_{P 0}^{3}}{v_{n 2}} \\
& a_{22}(\omega)=v_{n 1}^{2}\left[1-2 i k_{Q}\left(1+\epsilon_{Q 1}\right)\right]\left(1+2 \eta_{1}\right) \\
& a_{23}(\omega)=v_{P 0} v_{n 1}\left(1-2 i k_{Q}\right)-i k_{Q} \delta_{Q 1} \frac{v_{P 0}^{3}}{v_{n 1}} \\
& a_{33}(\omega)=v_{P 0}^{2}\left(1-2 i k_{Q}\right)
\end{aligned}
$$


with

$$
k_{Q}(\omega)=\operatorname{sgn}(\omega) \frac{A_{P 0}}{1-A_{P 0}^{2}}
$$

and

$$
\xi(\omega)=\sqrt{\frac{\left(1+2 \eta_{1}\right)\left(1+2 \eta_{2}\right)}{1+2 \eta_{3}}},
$$

where the argument $\omega$ of the quantities in the right-side of equations 11-13 is omitted. $i$ denotes the imaginary unit. $v_{P 0}$ denotes the vertical velocity in the nonattenuating reference medium $\left(A_{P 0}=0\right)$. The subscripts "1", "2" and "3" denote the $x$-, $y$ - and $z$-axes normal to the $[y, z],[x, z]$ and $[x, y]$ planes, respectively. $v_{n 1}$ and $v_{n 2}$ denote the NMO velocities in the $[y, z]$ plane of the reference medium. $\eta_{1}, \eta_{2}$ and $\eta_{3}$ denote the anellipticity parameters in the $[y, z],[x, z]$ and $[x, y]$ planes, respectively, of the reference medium. $A_{P 0}$ denotes the wavenumber-normalized attenuation coefficient of the vertically propagating $\mathrm{P}$-wave. $\epsilon_{1}$ and $\delta_{1}$ are the attenuation-anisotropy parameters defined in the $[y, z]$ plane. $\epsilon_{2}$ and $\delta_{2}$ are the attenuation-anisotropy parameters defined in the $[x, z]$ plane. $\delta_{3}$ are the attenuationanisotropy parameters in the $[x, y]$ plane. The definition of all these medium parameters is shown in Appendix A. Equation 13 is related to the parameter $\eta_{x y}$ defined in Stovas (2015).

We first take into account the derivation of the scalar wave equation. The dispersion relation can be obtained from the Christoffel equation for a viscoacoustic orthorhombic medium. Referring to Hao and Alkhalifah (2017a), the dispersion relation is written as

$$
\begin{aligned}
\omega^{6} & =\hat{A} \omega^{4} k_{1}^{2}+\hat{B} \omega^{4} k_{2}^{2}+\hat{C} \omega^{4} k_{3}^{2}+\hat{D} \omega^{2} k_{1}^{2} k_{2}^{2}+\hat{E} \omega^{2} k_{1}^{2} k_{3}^{2} \\
& +\hat{F} \omega^{2} k_{2}^{2} k_{3}^{2}+\hat{G} k_{1}^{2} k_{2}^{2} k_{3}^{2},
\end{aligned}
$$


with

$$
\begin{gathered}
\hat{A}=a_{11}, \\
\hat{B}=a_{22}, \\
\hat{C}=a_{33}, \\
\hat{D}=a_{12}^{2}-a_{11} a_{22}, \\
\hat{E}=a_{13}^{2}-a_{11} a_{33}, \\
\hat{F}=a_{23}^{2}-a_{22} a_{33}, \\
\hat{G}=-a_{11} a_{23}^{2}-a_{22} a_{13}^{2}-a_{33} a_{12}^{2} \\
+2 a_{12} a_{13} a_{23}+a_{11} a_{22} a_{33},
\end{gathered}
$$

where $k_{1}, k_{2}$ and $k_{3}$ denote the $x$-, $y$ - and $z$-components of the wavenumber vector, and the variable $\omega$ of the functions $\hat{A} \ldots \hat{G}$ and $a_{i j}$ is omitted for brevity.

We introduce a pseudo-pressure field $\hat{P}$ in the frequency-wavenumber domain. We multiply equation 14 by $\hat{P}$, utilize the inverse Fourier transform, and take account of the correspondence relation between equations 6 and 9. As a result, the viscoacoustic wave equation in the scalar form is written as

$$
\begin{aligned}
\frac{\partial^{6} P}{\partial t^{6}} & =A \odot \frac{\partial^{6} P}{\partial t^{4} \partial x^{2}}+B \odot \frac{\partial^{6} P}{\partial t^{4} \partial y^{2}}+C \odot \frac{\partial^{6} P}{\partial t^{4} \partial z^{2}}+D \odot \frac{\partial^{6} P}{\partial t^{2} \partial x^{2} \partial y^{2}} \\
& +E \odot \frac{\partial^{6} P}{\partial t^{2} \partial x^{2} \partial z^{2}}+F \odot \frac{\partial^{6} P}{\partial t^{2} \partial y^{2} \partial z^{2}}+G \odot \frac{\partial^{6} P}{\partial x^{2} \partial y^{2} \partial z^{2}}
\end{aligned}
$$


with

$$
\begin{gathered}
A=\phi_{11}, \\
B=\phi_{22}, \\
C=\phi_{33}, \\
D=\phi_{12} \odot \phi_{12}-\phi_{11} \odot \phi_{22}, \\
E=\phi_{13} \odot \phi_{13}-\phi_{11} \odot \phi_{33}, \\
F=\phi_{23} \odot \phi_{23}-\phi_{22} \odot \phi_{33}, \\
G=-\phi_{11} \odot \phi_{23} \odot \phi_{23}-\phi_{22} \odot \phi_{13} \odot \phi_{13}-\phi_{33} \odot \phi_{12} \odot \phi_{12} \\
+2 \phi_{12} \odot \phi_{13} \odot \phi_{23}+\phi_{11} \odot \phi_{22} \odot \phi_{33},
\end{gathered}
$$

where the argument $t$ is omitted for the funtions $A \ldots G$ and $\phi_{i j}, P=P(\mathbf{x}, t)$ denotes the pseudo-pressure wavefield in the time-space domain, and $\phi_{i j}$ denote the density-normalized second-order relaxation functions. The property 4 of the Riemann-Stieltjes convolution integral has been taken into account in equations 17.

We next take into account the derivation of the vector wave equations. Utilizing the equation of motion, the constitutive equation and the relation between the stress and the displacement, we derive the acoustic wave equations in the vector form as follows.

The wave equations in terms of the particle displacement and the normal stresses are given by

$$
\begin{aligned}
& \frac{\partial^{2} u_{x}}{\partial t^{2}}=\phi_{11} \odot \frac{\partial^{2} u_{x}}{\partial x^{2}}+\phi_{12} \odot \frac{\partial^{2} u_{y}}{\partial x \partial y}+\phi_{13} \odot \frac{\partial^{2} u_{z}}{\partial x \partial z}, \\
& \frac{\partial^{2} u_{y}}{\partial t^{2}}=\phi_{12} \odot \frac{\partial^{2} u_{x}}{\partial x \partial y}+\phi_{22} \odot \frac{\partial^{2} u_{y}}{\partial y^{2}}+\phi_{23} \odot \frac{\partial^{2} u_{z}}{\partial y \partial z}, \\
& \frac{\partial^{2} u_{z}}{\partial t^{2}}=\phi_{13} \odot \frac{\partial^{2} u_{x}}{\partial x \partial z}+\phi_{23} \odot \frac{\partial^{2} u_{y}}{\partial y \partial z}+\phi_{33} \odot \frac{\partial^{2} u_{z}}{\partial z^{2}},
\end{aligned}
$$


The wave equations in terms of the normal stresses are given by

$$
\begin{aligned}
& \frac{\partial^{2} \sigma_{x x}}{\partial t^{2}}=\phi_{11} \odot \frac{\partial^{2} \sigma_{x x}}{\partial x^{2}}+\phi_{12} \odot \frac{\partial^{2} \sigma_{y y}}{\partial y^{2}}+\phi_{13} \odot \frac{\partial^{2} \sigma_{z z}}{\partial z^{2}}, \\
& \frac{\partial^{2} \sigma_{y y}}{\partial t^{2}}=\phi_{12} \odot \frac{\partial^{2} \sigma_{x x}}{\partial x^{2}}+\phi_{22} \odot \frac{\partial^{2} \sigma_{y y}}{\partial y^{2}}+\phi_{23} \odot \frac{\partial^{2} \sigma_{z z}}{\partial z^{2}}, \\
& \frac{\partial^{2} \sigma_{z z}}{\partial t^{2}}=\phi_{13} \odot \frac{\partial^{2} \sigma_{x x}}{\partial x^{2}}+\phi_{23} \odot \frac{\partial^{2} \sigma_{y y}}{\partial y^{2}}+\phi_{33} \odot \frac{\partial^{2} \sigma_{z z}}{\partial z^{2}},
\end{aligned}
$$

where $u_{i}$ and $\sigma_{i i}$ denote the particle displacement components and the normal stress components, respectively. The detailed derivation of equations 18 and 19 is shown in Appendices $\mathrm{B}$ and $\mathrm{C}$.

The wave equations in terms of the momentum densities and the normal stresses are given by

$$
\begin{gathered}
\frac{\partial J_{x}}{\partial t}=\frac{\partial \sigma_{x x}}{\partial x}, \\
\frac{\partial J_{y}}{\partial t}=\frac{\partial \sigma_{y y}}{\partial y}, \\
\frac{\partial J_{z}}{\partial t}=\frac{\partial \sigma_{z z}}{\partial z}, \\
\frac{\partial \sigma_{x x}}{\partial t}=\phi_{11} \odot \frac{\partial J_{x}}{\partial x}+\phi_{12} \odot \frac{\partial J_{y}}{\partial y}+\phi_{13} \odot \frac{\partial J_{z}}{\partial z}, \\
\frac{\partial \sigma_{y y}}{\partial t}=\phi_{12} \odot \frac{\partial J_{x}}{\partial x}+\phi_{22} \odot \frac{\partial J_{y}}{\partial y}+\phi_{23} \odot \frac{\partial J_{z}}{\partial z}, \\
\frac{\partial \sigma_{z z}}{\partial t}=\phi_{13} \odot \frac{\partial J_{x}}{\partial x}+\phi_{23} \odot \frac{\partial J_{y}}{\partial y}+\phi_{33} \odot \frac{\partial J_{z}}{\partial z},
\end{gathered}
$$

where $\mathbf{J}=\left(J_{x}, J_{y}, J_{z}\right)^{T}$ denotes the momentum density $(\mathbf{J}=\rho \partial \mathbf{u} / \partial t)$ according to Auld (1973). The first three rows of equation 20 are obtained from the equation of motion by assuming the density to be constant. The last three rows in equation 20 are obtained from the constitutive equation.

If the density-normalized relaxation functions $\phi_{i j}$ are constant (i.e. independent of time), the viscoacoustic medium will become nonattenuating. Referring to the property 5 of the Riemann-Stieltjes convolution integral, all of the proposed viscoacoustic wave equations can reduce to the acoustic wave equations for orthorhombic anisotropy. In this case, the scalar 
wave equation 16 and the vector wave equations 19 and 20 reduce to equation 17 in Alkhalifah (2003) and equations 6 and 5 in Fowler and King (2011), respectively. For transverse isotropy with a vertical symmetry axis (VTI), the orthorhombic medium parameters $\left(v_{n 1}\right.$, $\eta_{1}, \epsilon_{Q 1}$ and $\left.\delta_{Q 1}\right)$ defined in the $[y, z]$ plane are identical to the parameters $\left(v_{n 2}, \eta_{2}, \epsilon_{Q 2}\right.$ and $\left.\delta_{Q 2}\right)$ defined in the $[x, z]$ plane, and the parameters $\left(\eta_{3}\right.$ and $\left.\delta_{Q 3}\right)$ defined in the $[x, y]$ plane vanish. Hence, the proposed wave equations can be simplified for transverse isotropy. Appendix D shows the viscoacoustic VTI wave equations.

\section{VISCOACOUSTIC ORTHORHOMBIC MODELS AND THE CORRESPONDING WAVE EQUATIONS}

Explicit expressions for the relaxation functions are needed to implement the wave equations shown in the previous section. We take into account the classic viscoacoustic models, i.e., Kelvin-Voigt model, the Maxwell model, the SLS model, and the Kjartansson model. A detailed description of these models can be found in Carcione (2015).

For these models, we assume that the parameters in the modified Alkhalifah's (2003) and Zhu and Tsvankin's (2007) notations are known at a selected reference angular frequency $\left(\omega_{c}>0\right)$, and denote them by putting a bar over them, e.g. $\bar{v}_{P 0}=v_{P 0}\left(\omega_{c}\right)$ and $\bar{A}_{P 0}=$ $A_{P 0}\left(\omega_{c}\right)$. The reference frequency corresponding to $\omega_{c}$ is denoted by $f_{c}$. As illustrated in equation 11, the density-normalized stiffness coefficients can be expressed in terms of the parameters of the modified Alkhalifah's (2003) and Zhu and Tsvankin's (2007) notations. The density-normalized stiffness coefficients at the reference angular frequency are denoted by $\bar{a}_{i j}=\bar{a}_{i j}^{R}-i \bar{a}_{i j}^{I}$. The elements of the quality factor matrix are denoted by $\bar{Q}_{i j}=$

$\bar{a}_{i j}^{R} / \bar{a}_{i j}^{I}$. Using these parameters with a specified viscoacoustic model at the reference angular 
frequency, we can determine the density-normalized relaxation functions $\phi_{i j}$ and the densitynormalized stiffness coefficients $a_{i j}$ as a function of frequency. Substituting the densitynormalized relaxation functions for a specified viscoacoustic model into the vector wave equations 19, we may derive the wave equations in differential form. The differential form of the other vector wave equations can be obtained in a similar way.

The classic viscoacoustic models and their corresponding wave equations are summarized as follows.

\section{The Kelvin-Voigt model}

The density-normalized relaxation functions and stiffness coefficients for the Kelvin-Voigt model are given by

$$
\begin{gathered}
\phi_{i j}(t)=\eta_{i j}^{(1)} \delta(t)+m_{i j}^{(1)} h(t), \\
a_{i j}(\omega)=m_{i j}^{(1)}-i \omega \eta_{i j}^{(1)},
\end{gathered}
$$

with

$$
\begin{gathered}
m_{i j}^{(1)}=\bar{a}_{i j}^{R}, \\
\eta_{i j}=\frac{\bar{a}_{i j}^{R}}{2 \pi f_{c} \bar{Q}_{i j}},
\end{gathered}
$$

where $\delta(t)$ denotes the Dirac delta function, $h(t)$ denotes the Heaviside step function, $m_{i j}^{(1)}$

denote the density-normalized elastic stiffness coefficients, and $\eta_{i j}^{(1)}$ denote the densitynormalized viscous coefficients. 
The wave equations for the Kelvin-Voigt model are given by

$$
\begin{aligned}
& \frac{\partial^{2} \sigma_{x x}}{\partial t^{2}}=\left(m_{11}^{(1)}+\eta_{11}^{(1)} \frac{\partial}{\partial t}\right) \frac{\partial^{2} \sigma_{x x}}{\partial x^{2}}+\left(m_{12}^{(1)}+\eta_{12}^{(1)} \frac{\partial}{\partial t}\right) \frac{\partial^{2} \sigma_{y y}}{\partial y^{2}}+\left(m_{13}^{(1)}+\eta_{13}^{(1)} \frac{\partial}{\partial t}\right) \frac{\partial^{2} \sigma_{z z}}{\partial z^{2}}, \\
& \frac{\partial^{2} \sigma_{y y}}{\partial t^{2}}=\left(m_{12}^{(1)}+\eta_{12}^{(1)} \frac{\partial}{\partial t}\right) \frac{\partial^{2} \sigma_{x x}}{\partial x^{2}}+\left(m_{22}^{(1)}+\eta_{22}^{(1)} \frac{\partial}{\partial t}\right) \frac{\partial^{2} \sigma_{y y}}{\partial y^{2}}+\left(m_{23}^{(1)}+\eta_{23}^{(1)} \frac{\partial}{\partial t}\right) \frac{\partial^{2} \sigma_{z z}}{\partial z^{2}} \\
& \frac{\partial^{2} \sigma_{z z}}{\partial t^{2}}=\left(m_{13}^{(1)}+\eta_{13}^{(1)} \frac{\partial}{\partial t}\right) \frac{\partial^{2} \sigma_{x x}}{\partial x^{2}}+\left(m_{23}^{(1)}+\eta_{23}^{(1)} \frac{\partial}{\partial t}\right) \frac{\partial^{2} \sigma_{y y}}{\partial y^{2}}+\left(m_{33}^{(1)}+\eta_{33}^{(1)} \frac{\partial}{\partial t}\right) \frac{\partial^{2} \sigma_{z z}}{\partial z^{2}}
\end{aligned}
$$

\section{The Maxwell model}

The density-normalized relaxation functions and stiffness coefficients for the Maxwell model are given by

$$
\begin{aligned}
\phi_{i j}(t) & =m_{i j}^{(2)} e^{-m_{i j}^{(2)} t / \eta_{i j}^{(2)}} h(t), \\
a_{i j}(\omega) & =\left(\frac{1}{m_{i j}^{(2)}}+\frac{i}{\omega \eta_{i j}^{(2)}}\right)^{-1}
\end{aligned}
$$

with

$$
\begin{gathered}
m_{i j}^{(2)}=\bar{a}_{i j}^{R}\left(1+\frac{1}{\bar{Q}_{i j}^{2}}\right), \\
\eta_{i j}^{(2)}=\frac{\bar{a}_{i j}^{R}}{2 \pi f_{c}}\left(\bar{Q}_{i j}+\frac{1}{\bar{Q}_{i j}}\right),
\end{gathered}
$$

where $m_{i j}^{(2)}$ denote the density-normalized elastic stiffness coefficients as $\omega \rightarrow \infty, \eta_{i j}^{(2)}$ denote the density-normalized viscous coefficients.

The wave equations for the Maxwell model are given by

$$
\begin{aligned}
& \frac{\partial^{2} \sigma_{x x}}{\partial t^{2}}=m_{11}^{(2)} \frac{\partial^{2} \sigma_{x x}}{\partial x^{2}}+m_{12}^{(2)} \frac{\partial^{2} \sigma_{y y}}{\partial y^{2}}+m_{13}^{(2)} \frac{\partial^{2} \sigma_{z z}}{\partial z^{2}}-\sum_{j=1}^{3} r_{1 j}, \\
& \frac{\partial^{2} \sigma_{y y}}{\partial t^{2}}=m_{12}^{(2)} \frac{\partial^{2} \sigma_{x x}}{\partial x^{2}}+m_{22}^{(2)} \frac{\partial^{2} \sigma_{y y}}{\partial y^{2}}+m_{23}^{(2)} \frac{\partial^{2} \sigma_{z z}}{\partial z^{2}}-\sum_{j=1}^{3} r_{2 j}, \\
& \frac{\partial^{2} \sigma_{z z}}{\partial t^{2}}=m_{13}^{(2)} \frac{\partial^{2} \sigma_{x x}}{\partial x^{2}}+m_{23}^{(2)} \frac{\partial^{2} \sigma_{y y}}{\partial y^{2}}+m_{33}^{(2)} \frac{\partial^{2} \sigma_{z z}}{\partial z^{2}}-\sum_{j=1}^{3} r_{3 j},
\end{aligned}
$$

where the auxiliary variables $r_{i j}$ satisfy

$$
\eta_{i j}^{(2)} \frac{\partial r_{i j}}{\partial t}=\left(m_{i j}^{(2)}\right)^{2} \frac{\partial^{2} \sigma_{j j}}{\partial x_{j}^{2}}-m_{i j}^{(2)} r_{i j}
$$


Here, the subscripts " $i$ " and " $j$ " are taken as 1,2 and 3 or $x, y$ and $z$, and the repeated indices do not satisfy the Einstein summation convention.

\section{The SLS model}

The density-normalized relaxation functions and stiffness coefficients for the SLS model are given by

$$
\begin{gathered}
\phi_{i j}(t)=m_{i j}^{(3)}\left[1-\left(1-\frac{\tau_{i j}}{\tau_{\sigma}}\right) e^{-t / \tau_{\sigma}}\right] h(t), \\
a_{i j}(\omega)=m_{i j}^{(3)} \frac{1-i \omega \tau_{i j}}{1-i \omega \tau_{\sigma}}
\end{gathered}
$$

with

$$
\begin{gathered}
m_{i j}^{(3)}=\bar{a}_{i j}^{R}\left(1-\frac{1}{\bar{Q}_{i j}}\right), \\
\tau_{i j}=\tau_{\sigma} \frac{\bar{Q}_{i j}+1}{\bar{Q}_{i j}-1}, \\
\tau_{\sigma}=\frac{1}{2 \pi f_{c}},
\end{gathered}
$$

where equation 36 follows Bai et al. (2017), $m_{i j}^{(3)}$ denote the density-normalized elastic stiffness coefficients corresponding to $t \rightarrow \infty$ or $\omega \rightarrow 0, \tau_{i j}$ denote the strain relaxation time, and $\tau_{\sigma}$ denotes the stress relaxation time.

The wave equations for the SLS model are given by

$$
\begin{aligned}
\frac{\partial^{2} \sigma_{x x}}{\partial t^{2}} & =\zeta_{11} m_{11}^{(3)} \frac{\partial^{2} \sigma_{x x}}{\partial x^{2}}+\zeta_{12} m_{12}^{(3)} \frac{\partial^{2} \sigma_{y y}}{\partial y^{2}}+\zeta_{13} m_{13}^{(3)} \frac{\partial^{2} \sigma_{z z}}{\partial z^{2}}-w_{1}, \\
\frac{\partial^{2} \sigma_{y y}}{\partial t^{2}} & =\zeta_{12} m_{12}^{(3)} \frac{\partial^{2} \sigma_{x x}}{\partial x^{2}}+\zeta_{22} m_{22}^{(3)} \frac{\partial^{2} \sigma_{y y}}{\partial y^{2}}+\zeta_{23} m_{23}^{(3)} \frac{\partial^{2} \sigma_{z z}}{\partial z^{2}}-w_{2}, \\
\frac{\partial^{2} \sigma_{z z}}{\partial t^{2}} & =\zeta_{13} m_{13}^{(3)} \frac{\partial^{2} \sigma_{x x}}{\partial x^{2}}+\zeta_{23} m_{23}^{(3)} \frac{\partial^{2} \sigma_{y y}}{\partial y^{2}}+\zeta_{33} m_{33}^{(3)} \frac{\partial^{2} \sigma_{z z}}{\partial z^{2}}-w_{3},
\end{aligned}
$$


where $w_{i}$ are the auxiliary variables given by

$$
\begin{aligned}
& \frac{\partial w_{1}}{\partial t}=n_{11} \frac{\partial^{2} \sigma_{x x}}{\partial x^{2}}+n_{12} \frac{\partial^{2} \sigma_{y y}}{\partial y^{2}}+n_{13} \frac{\partial^{2} \sigma_{z z}}{\partial z^{2}}-\frac{1}{\tau_{\sigma}} w_{1}, \\
& \frac{\partial w_{2}}{\partial t}=n_{12} \frac{\partial^{2} \sigma_{x x}}{\partial x^{2}}+n_{22} \frac{\partial^{2} \sigma_{y y}}{\partial y^{2}}+n_{23} \frac{\partial^{2} \sigma_{z z}}{\partial z^{2}}-\frac{1}{\tau_{\sigma}} w_{2}, \\
& \frac{\partial w_{3}}{\partial t}=n_{13} \frac{\partial^{2} \sigma_{x x}}{\partial x^{2}}+n_{23} \frac{\partial^{2} \sigma_{y y}}{\partial y^{2}}+n_{33} \frac{\partial^{2} \sigma_{z z}}{\partial z^{2}}-\frac{1}{\tau_{\sigma}} w_{3},
\end{aligned}
$$

and $n_{i j}=\left(\zeta_{i j}-1\right) m_{i j}^{(3)} / \tau_{\sigma}$ and $\zeta_{i j}=\tau_{i j} / \tau_{\sigma}$.

\section{The Kjartansson model}

The density-normalized relaxation functions and stiffness coefficients for the Kjartansson model are given by

$$
\begin{gathered}
\phi_{i j}(t)=\frac{m_{i j}^{(4)}}{\Gamma\left(1-2 \gamma_{i j}\right)}\left(\frac{t}{t_{c}}\right)^{-2 \gamma_{i j}} h(t), \\
a_{i j}(\omega)=m_{i j}^{(4)}\left(\frac{-i \omega}{\omega_{c}}\right)^{2 \gamma_{i j}}
\end{gathered}
$$

with

$$
\begin{gathered}
\gamma_{i j}=\frac{1}{\pi} \arctan \left(\frac{1}{\bar{Q}_{i j}}\right), \\
m_{i j}^{(4)}=\bar{a}_{i j}^{R} \sec \left(\pi \gamma_{i j}\right), \\
\omega_{c}=\frac{1}{t_{c}}=2 \pi f_{c},
\end{gathered}
$$

where $\Gamma$ (.) denotes the gamma function (Arfken and Weber, 2001), $m_{i j}^{(4)}$ denote the densitynormalized elastic stiffness coefficients corresponding to $\gamma_{i j} \rightarrow 0$ for $t>0$, and $t_{c}$ denotes the reference time (Kjartansson, 1979).

The wave equations for the Kjartansson model are given by

$$
\begin{aligned}
& \frac{\partial^{2} \sigma_{x x}}{\partial t^{2}}=\tilde{m}_{11}^{(4)} D_{t}^{2 \gamma_{11}} \frac{\partial^{2} \sigma_{x x}}{\partial x^{2}}+\tilde{m}_{12}^{(4)} D_{t}^{2 \gamma_{12}} \frac{\partial^{2} \sigma_{y y}}{\partial y^{2}}+\tilde{m}_{13}^{(4)} D_{t}^{2 \gamma_{13}} \frac{\partial^{2} \sigma_{z z}}{\partial z^{2}} \\
& \frac{\partial^{2} \sigma_{y y}}{\partial t^{2}}=\tilde{m}_{12}^{(4)} D_{t}^{2 \gamma_{12}} \frac{\partial^{2} \sigma_{x x}}{\partial x^{2}}+\tilde{m}_{22}^{(4)} D_{t}^{2 \gamma_{22}} \frac{\partial^{2} \sigma_{y y}}{\partial y^{2}}+\tilde{m}_{23}^{(4)} D_{t}^{2 \gamma_{23}} \frac{\partial^{2} \sigma_{z z}}{\partial z^{2}} \\
& \frac{\partial^{2} \sigma_{z z}}{\partial t^{2}}=\tilde{m}_{13}^{(4)} D_{t}^{2 \gamma_{13}} \frac{\partial^{2} \sigma_{x x}}{\partial x^{2}}+\tilde{m}_{23}^{(4)} D_{t}^{2 \gamma_{23}} \frac{\partial^{2} \sigma_{y y}}{\partial y^{2}}+\tilde{m}_{33}^{(4)} D_{t}^{2 \gamma_{33}} \frac{\partial^{2} \sigma_{z z}}{\partial z^{2}}
\end{aligned}
$$


where $\tilde{m}_{i j}^{(4)}=m_{i j}^{(4)} / \omega_{c}^{2 \gamma_{i j}}$ and $D_{t}^{2 \gamma_{i j}}$ denote the fractional temporal derivative of orders $2 \gamma_{i j}$, given by (Mainardi, 2010; Carcione, 2015)

$$
D_{t}^{2 \gamma_{i j}} g(t)=\frac{1}{\Gamma\left(1-2 \gamma_{i j}\right)} \int_{0}^{t} \frac{\dot{g}(\tau)}{(t-\tau)^{2 \gamma_{i j}}} d \tau
$$

Here, $g$ denotes the function of time, the dot on $\dot{g}$ denotes the first derivative, and $\gamma_{i j}$ are assumed to satisfy $0<\gamma_{i j}<0.5$, which is valid for real attenuating rocks. A detailed description of fractional derivatives can be found in Podlubny (1998). In the derivation of equations 44 from equations 19 with equation 39, we have taken into account equation 8 with the property 4 .

\section{THE ACOUSTIC RADIATION FROM A POINT SOURCE}

We put a point-source term $f(\mathbf{x}, t)=\frac{\partial^{4} S(t)}{\partial t^{4}} \delta(x) \delta(y) \delta(z)$ into the right side of the scalar wave equation 16, where $S(t)$ is a causal signal, $\delta(\cdot)$ denotes the Dirac delta function. We shall explain later why we adopt the fourth-order derivative of the signal to describe a source wavelet. Applying Fourier transform to equation 16 over $x, y, z$ and $t$, we obtain the point-source solution in the frequency-wavenumber domain,

$$
\hat{P}(\mathbf{k}, \omega)=\frac{\omega^{4} \hat{S}(\omega)}{H(\mathbf{k}, \omega)}
$$

with

$$
\begin{aligned}
H(\mathbf{k}, \omega) & =\hat{A}(\omega) \omega^{4} k_{1}^{2}+\hat{B}(\omega) \omega^{4} k_{2}^{2}+\hat{C}(\omega) \omega^{4} k_{3}^{2}+\hat{D}(\omega) \omega^{2} k_{1}^{2} k_{2}^{2}+\hat{E}(\omega) \omega^{2} k_{1}^{2} k_{3}^{2} \\
& +\hat{F}(\omega) \omega^{2} k_{2}^{2} k_{3}^{2}+\hat{G}(\omega) k_{1}^{2} k_{2}^{2} k_{3}^{2}-\omega^{6}
\end{aligned}
$$

where $\mathbf{k}=\left(k_{1}, k_{2}, k_{3}\right)^{T}$ denotes the wavenumber vector in the Cartesian $(x, y, z)$ system.

The inverse Fourier transform of equation 46 over $k_{1}, k_{2}$ and $k_{3}$ leads to the point-source 
solution in the frequency-space domain,

$$
\hat{P}(\mathbf{x}, \omega)=\frac{1}{8 \pi^{3}} \int_{-\infty}^{\infty} \int_{-\infty}^{\infty} \int_{-\infty}^{\infty} \frac{\omega^{4} \hat{S}(\omega)}{H(\mathbf{k}, \omega)} e^{i\left(k_{1} x+k_{2} y+k_{3} z\right)} \mathrm{d} k_{1} \mathrm{~d} k_{2} \mathrm{~d} k_{3} .
$$

We adopt the Buckward's (1959) method to derive an asymptotic expression for the integrals in equation 48. The Cartesian coordinate $(x, y, z)$ of the observation point is identical to the spherical coordinate $(r, \alpha, \beta)$, where $r$ denotes the radial distance between the observation point and the coordinate origin, $\alpha$ denotes the polar angle measured from the positive $z$-axis, $\beta$ denotes the azimuth measured from the positve $x$-axis in the $[x, y]$ plane. We now perform a rotation of the Cartesian coordinate system so that the observation point is located in the positve $z^{\prime}$-axis of the new Cartesian coordinate system $\left(x^{\prime}, y^{\prime}, z^{\prime}\right)$. Various ways can be adopted to realize this aim. For example, we use the following rotation

$$
\mathbf{x}=\mathbf{M x}^{\prime}
$$

where $\mathbf{x}$ and $\mathbf{x}^{\prime}$ denote the coordinates of the observation point in the Cartesian $(x, y, z)$ and $\left(x^{\prime}, y^{\prime}, z^{\prime}\right)$ coordinate systems, respectively, and $\mathbf{M}$ denotes the orthogonal transformation matrix given by

$$
\mathbf{M}=\left(\begin{array}{ccc}
\cos \beta \cos \alpha & -\sin \beta & \cos \beta \sin \alpha \\
\sin \beta \cos \alpha & \cos \beta & \sin \beta \sin \alpha \\
-\sin \alpha & 0 & \cos \alpha
\end{array}\right) .
$$

The first, second and third columns of the matrix $\mathbf{M}$ describe the base vectors along the $x^{\prime}-, y^{\prime}$ - and $z^{\prime}$-axes in the Cartesian coordinate system $(x, y, z)$. The Cartesian coordinate systems before and after the rotation satisfy the right-hand rule. Applying the coordinate rotation, the integrals in equation 48 reduce to

$$
\hat{P}(\mathbf{x}, \omega)=\frac{1}{8 \pi^{3}} \int_{-\infty}^{\infty} \int_{-\infty}^{\infty} \int_{-\infty}^{\infty} \frac{\omega^{4} \hat{S}(\omega)}{H^{\prime}\left(\mathbf{k}^{\prime}, \omega\right)} e^{i k_{3}^{\prime} r} \mathrm{~d} k_{1}^{\prime} \mathrm{d} k_{2}^{\prime} \mathrm{d} k_{3}^{\prime},
$$


where $\mathbf{k}^{\prime}=\left(k_{1}^{\prime}, k_{2}^{\prime}, k_{3}^{\prime}\right)^{T}$ denotes the wavenumber vector in the Cartesian coordinate system $\left(x^{\prime}, y^{\prime}, z^{\prime}\right)$, and the function $H^{\prime}$ is given by

$$
H^{\prime}\left(\mathbf{k}^{\prime}, \omega\right)=H\left(\mathbf{M k}^{\prime}, \omega\right)
$$

The integral with respect to $k_{3}^{\prime}$ in equation 51 can be calculated by the residue theorem. The residues correspond to the roots of the equation $H^{\prime}\left(\mathbf{k}^{\prime}, \omega\right)=0$ about $k_{3}^{\prime}$. The equation generally is a six-order algebric equation with respect to $k_{3}^{\prime}$. Two roots of the equation correspond to the up- and down-going $\mathrm{P}$-waves and the remaining roots correspond to the up- and down-going pseudo $S_{1}$ - and $S_{2}$-waves. The pseudo $S_{1^{-}}$and $S_{2}$-waves do not have the polarization behaviors of the true shear waves, and their velocities are much smaller than those of the true shear waves in an anisotropic media. Hence, the pseudo $S_{1^{-}}$and $S_{2}$-waves are useless in seismic exploration, and we may concern ourselves with only the P-wave radiation. We use $\breve{k}_{3}^{\prime}$ to denote the $\mathrm{P}$-wave root in the upper $\left(\operatorname{Im}\left(k_{3}^{\prime}\right)>0\right)$ of the complex $k_{3}^{\prime}$-plane. To calculate the integral along the real $k_{3}^{\prime}$ axis in equation 51 , we may construct a contour in the complex $k_{3}^{\prime}$-plane. Since the observation point is located in the positive $z^{\prime}$-axis of the new Cartesian coordinate system, we may let the contour be composed of the real $k_{3}^{\prime}$ axis and the infinite-radius semicircle in the upper half-space of the complex $k_{3}^{\prime}$-plane and hence the contour includes the pole $\breve{k}_{3}^{\prime}\left(k_{1}^{\prime}, k_{2}^{\prime}\right)$. Referring to residue theorem, equation 51 is rewritten as

$$
\hat{P}(\mathbf{x}, \omega)=\frac{i}{4 \pi^{2}} \int_{-\infty}^{\infty} \int_{-\infty}^{\infty}\left\{\frac{\omega^{4} \hat{S}(\omega)}{\partial H^{\prime}\left(\mathbf{k}^{\prime}, \omega\right) / \partial k_{3}^{\prime}} e^{i k_{3}^{\prime} r}\right\}_{k_{3}^{\prime}=\breve{k}_{3}^{\prime}\left(k_{1}^{\prime}, k_{2}^{\prime}\right)} \mathrm{d} k_{1}^{\prime} \mathrm{d} k_{2}^{\prime} .
$$

We next attempt to derive the asymptotic approximation for the point-source solution. We substitute $p_{i}^{\prime}=k_{i}^{\prime} / \omega$ into equation 53 to change the variables in the integral, where $p_{i}^{\prime}$ denote the slowness components in the Cartesian coordinate system $\left(x^{\prime}, y^{\prime}, z^{\prime}\right)$. The point source 
solution is rewritten as

$$
\hat{P}(\mathbf{x}, \omega)=\frac{i \omega}{4 \pi^{2}} \int_{-\infty}^{\infty} \int_{-\infty}^{\infty}\left\{\frac{\hat{S}(\omega)}{\partial I^{\prime}\left(\mathbf{p}^{\prime}, \omega\right) / \partial p_{3}^{\prime}} e^{i \omega p_{3}^{\prime} r}\right\}_{p_{3}^{\prime}=\breve{p}_{3}^{\prime}\left(p_{1}^{\prime}, p_{2}^{\prime}\right)} \mathrm{d} p_{1}^{\prime} \mathrm{d} p_{2}^{\prime} .
$$

where $\breve{p}_{3}^{\prime}=\breve{k}_{z}^{\prime} / \omega$ is a function of the horizontal slowness components $p_{1}^{\prime}$ and $p_{2}^{\prime}$ and the function $I$ is given by

$$
I^{\prime}\left(\mathbf{p}^{\prime}, \omega\right)=H\left(\mathbf{M p}^{\prime} \omega, \omega\right) / \omega^{6}
$$

It is noteworthy that equation $I^{\prime}\left(\mathbf{p}^{\prime}, \omega\right)=0$ is the acoustic eikonal equation in an attenuating orthorhombic medium with tilted symmetry axes (Hao and Alkhalifah, 2017a).

We adopt the steepest descent method to evaluate the complex integrals in equation 54 . The saddle point is determined by

$$
\left[\frac{\partial \breve{p}_{3}^{\prime}\left(p_{1}^{\prime}, p_{2}^{\prime}\right)}{\partial p_{1}^{\prime}}\right]_{\tilde{p}_{1}^{\prime}, \tilde{p}_{2}^{\prime}}=0, \quad\left[\frac{\partial \breve{p}_{3}^{\prime}\left(p_{1}^{\prime}, p_{2}^{\prime}\right)}{\partial p_{2}^{\prime}}\right]_{\tilde{p}_{1}^{\prime}, \tilde{p}_{2}^{\prime}}=0
$$

where $\tilde{p}_{1}^{\prime}$ and $\tilde{p}_{2}^{\prime}$ denote the solution of equation 56 . The vertical slowness component is approximated by a second-order Taylor expansion at the saddle point,

$$
\breve{p}_{3}^{\prime}\left(p_{1}^{\prime}, p_{2}^{\prime}\right) \approx \tilde{p}_{3}^{\prime}+\frac{1}{2} \sum_{i, j=1}^{2} \frac{\partial^{2} \tilde{p}_{3}^{\prime}}{\partial \tilde{p}_{i}^{\prime} \partial \tilde{p}_{j}^{\prime}}\left(p_{i}^{\prime}-\tilde{p}_{i}^{\prime}\right)\left(p_{j}^{\prime}-\tilde{p}_{j}^{\prime}\right)
$$

where $\tilde{p}_{3}^{\prime}=\breve{p}_{3}^{\prime}\left(\tilde{p}_{1}^{\prime}, \tilde{p}_{2}^{\prime}\right)$ denotes the vertical slowness component at the saddle point. The slowness $\tilde{\mathbf{p}}^{\prime}=\left(\tilde{p}_{1}^{\prime}, \tilde{p}_{2}^{\prime}, \tilde{p}_{3}^{\prime}\right)$ describes the P-wave slowness corresponding to the observation direction $(\alpha, \beta)$. The slowness $\tilde{\mathbf{p}}^{\prime}$ is determined uniquely for a given observation direction.

In the following, we would omit arguments like $\omega$ and $\mathbf{x}$ of a function at the right side of an obtained equation for brevity. The substitution of equation 57 into equation 54 and evaluation of the integrals by the steepest descent method (Bleistein, 2012) leads to the point-source acoustic asymptotic solution,

$$
\hat{P}(\mathbf{x}, \omega) \approx \frac{1}{2 \pi r} \frac{1}{\sqrt{|K|}} \frac{\hat{S}}{\left|\partial \tilde{I}^{\prime} / \partial \tilde{p}_{3}^{\prime}\right|} \exp \left(-\omega A_{\text {ray }} r\right) \exp \left(i \omega \frac{r}{V_{\text {ray }}}-i \nu\right), \quad \omega \geq 0
$$


with

$$
\begin{gathered}
K=\operatorname{det}\left[\frac{\partial^{2} \tilde{p}_{3}^{\prime}}{\partial \tilde{p}_{i}^{\prime} \partial \tilde{p}_{j}^{\prime}}\right]=\frac{\partial^{2} \tilde{p}_{3}^{\prime}}{\partial \tilde{p}_{1}^{\prime 2}} \frac{\partial^{2} \tilde{p}_{3}^{\prime}}{\partial \tilde{p}_{2}^{\prime 2}}-\left(\frac{\partial^{2} \tilde{p}_{3}^{\prime}}{\partial \tilde{p}_{1}^{\prime} \partial \tilde{p}_{2}^{\prime}}\right)^{2}, \\
\nu=\arg \left(\frac{\partial I^{\prime}}{\partial \tilde{p}_{3}^{\prime}}\right)+\frac{1}{2} \arg (K), \\
V_{\text {ray }}=\frac{v_{R}^{2}+v_{I}^{2}}{v_{R}}, \quad A_{\text {ray }}=-\frac{v_{I}}{v_{R}^{2}+v_{I}^{2}},
\end{gathered}
$$

where $r$ denotes the radial distance between the observation position and the coordinate origin, the expression for $\tilde{I}^{\prime}=I^{\prime}\left(\tilde{\mathbf{p}}^{\prime}, \omega\right)$ can be obtained from equation 55 with equations 47 and 50, $K$ denotes the Gaussian curvature of the slowness surface at the saddle point, $V_{\text {ray }}$ and $A_{\text {ray }}$ denote the ray velocity and attenuation, and $v_{R}+i v_{I}=1 / \tilde{p}_{3}^{\prime}$ denotes the complex group velocity (Vavryčuk, 2007b). It is noteworthy that $v_{R}$ is always positive, and $v_{I}$ is negative for $\omega>0$ and positive for $\omega<0$, respectively.

Equation 58 describes the point-source solution for nonnegative frequencies $(\omega \geq 0)$. The solution for negative frequencies $(\omega<0)$ is obtained by taking the complex conjugate of equation 58. In the beginning of this section, we take the fourth-order derivative of the signal $S(t)$ as the source wavelet. Otherwise, equation 58 will include a term $1 / \omega^{4}$, which causes singularity at $\omega=0$. Referring to equation 2 , the time-domain point-source solution $P(\mathbf{x}, t)$ can be obtained by the inverse Fourier transform of $\hat{P}(\mathbf{x}, \omega)$ over $\omega$.

\section{NUMERICAL EXAMPLES}

In the first example, we would compare the point-source radiations from different models. We take into account the the Kelvin-Voigt model, the Maxwell model, the SLS model and the Kjartansson model. For brevity, we call these models the frequency-dependent viscoacoustic models. In addition, we also utilize a nonattenuating model and a frequencyindependent viscoacoustic model, where the term "frequency-independent viscoacoustic" 
means that the stiffness coefficients are complex-valued but do not vary with frequency.

For all of the frequency-dependent viscoacoustic models, we set the reference frequency as $f_{c}=40 \mathrm{~Hz}$. Utilizing equation 11 , we calculate the density-normalized stiffness coefficients at the reference frequency from the medium parameters shown in Table 1. For any other frequency, the density-normalized stiffness coefficients are determined from equations 22, 27, 33 and 40 for the Kelvin-Voigt model, the Maxwell model, the SLS model and the Kjartansson model, respectively. The frequency-independent viscoacoustic model is described by the medium parameters in Table 1 . The nonattenuating model is described by the velocity-related parameters $\left(\bar{v}_{P 0}, \bar{v}_{n 1}, \bar{v}_{n 2}, \bar{\eta}_{1}, \bar{\eta}_{2}\right.$ and $\left.\bar{\eta}_{3}\right)$ in Table 1.

We take into account the scalar viscoacoustic orthorhombic wave equation 16 . The timedomain point source wavelet is obtain by taking the fourth order derivative of the Ricker wavelet $S(t)$ in Figure 1. We implement equation 58 to compute the frequency-domain point-source radiations for all of the considered models. The time-domain point-source radiation is obtained by the inverse Fourier transform over the angular frequency. Figure 2 compares the point-source radiations for the viscoacoustic models. All of the waveforms excited by the point source are similar to a Ricker wavelet. The comprehensive effect of dispersion and frequency-dependent attenuation distorts the early- and late-arrival troughs of these waveforms. For the frequency-independent viscoacoustic model, the dispersion, the attenuation coefficient and the geometrical spreading do not vary with frequency. As illustrated by the dashed black lines in Figure 2, the early- and late-arrival troughs of the waveforms have the same amplitude in the frequency-independent viscoacoustic model. For other viscoacoustic models, the amplitudes of the early-arrival troughs are larger than those of the late-arrival troughs. This behavior is most significant for the SLS model but least significant for the Kelvin-Voigt model. Figure 3 shows that the waveforms from 
the SLS model is similar to those from the Kjartansson model. It is predictable that combining multiple SLS elements may approximate the Kjartansson model. In fact, Bai and Tsvankin (2016) demonstrated that the generalized SLS model can be used as an alternative to simulate the constant-Q wave propagation in viscoelastic anisotropic media. Figure 4 compares the waveforms from the nonattenuating model and the viscoacoustic models. The wave amplitudes from the nonattenuating orthorhombic model is much larger than those from the viscoacoustic models. It indicates that attenuation is an important factor causing the decay of wave amplitudes in a viscoacoustic medium.

In the second example, we would investigate the effect of the attenuation-anisotropy parameters (i.e. $\epsilon_{Q 1}, \delta_{Q 1}, \epsilon_{Q 2}, \delta_{Q 2}$ and $\delta_{Q 3}$ ) at the reference frequency on the waveforms in a homogeneous viscoacoustic orthorhombic model. We use the same point source as in the first example. Figure 5 compares the point-source radiations from the SLS model with anisotropic attenuation coefficients and the corresponding model with isotropic attenuation coefficients. The attenuation-anisotropy parameters affect the waveforms in the directions deviated from the $z$-axis, and this influence is most significant in the directions close to the $x$ - and $y$-axes. According to Zhu and Tsvankin (2007), the effect of the attenuationanisotropy parameters on the attenuation generally increases with the deviation of the propagation directions from the $z$-axis. For the SLS model with isotropic attenuation coefficients, the wave amplitude still varies with the wave propagation direction. Since the attenuation coefficient is isotropic in this case, it implies that the velocity anisotropy causes the wave amplitude anisotropy. By comparing the wave arrival times from the wavefroms in these two models, we find that the attenuation-anisotropy parameters do not affect the wave traveltimes. Hence, it is impossible to estimate the attenuation anisotropy from the traveltime data picked from a seismogram. 


\section{DISCUSSION}

We present the scalar and vector viscoacoustic wave equations for orthorhombic anisotropy with the symmetry planes orthogonal to the Cartesian coordinate axes. Utilizing coordinate rotations, these viscoacoustic wave equations can be extended to the case of tilted TI and orthorhombic anisotropies.

The proposed viscoacoustic wave equations $(16,18,19$ and 20) are naturally classified as the integral-differential equation. Directly solving these viscoacoustic wave equation is computationally costly due to the time convolution. In the section "viscoacoustic orthorhombic models and the corresponding wave equations", we obtain the differential form of the vector viscoacoustic wave equations 19 for the classic viscoacoustic models (i.e. the Kelvin-Voigt model, the Maxwell model, the SLS model and the Kjartansson model). As mentioned in that section, the other vector viscoacoustic wave equations can also be rewritten into the differential form for these viscoacoustic models. In fact, these vector wave equations in differential form are generally easy to solve. Only the wave equations 44 for the Kjartansson model are relatively complicated because of the fractional derivatives with respect to time. As suggested by Carcione et al. (2002), however, the fractional derivatives can be calculated by the Grunwald-Letnikov approximation and the central-difference approximation. The wave equations 44 can be solved numerically by analogy with Carcione et al. (2002) and Zhu (2017).

Except for the viscoacoustic models with power-law type relaxation functions (e.g. the Kjartansson model), it is generally difficult to obtain the differential form of the scalar viscoacoustic wave equation 16 . As illustrated in equation 16 with equation 17, the scalar viscoacoustic wave equation involves the multiple Riemann-Stieltjes convolution integral. For 
the Kelvin-Voigt model, the Maxwell model and the SLS model, it is not easy to introduce auxiliary variables to transform the scalar viscoacoustic wave equation into its differential form. For the power-law type models such as the Kjartansson model, the Riemann-Stieltjes convolution integral can be written as a fractional derivative multiplied by a constant coefficient, as illustrated in the wave equations 44. Hence, a multiple Riemann-Stieltjes convolution integral can be expressed in terms of a product of the fractional derivatives corresponding to each single integral, which can be combined into a single fractional derivative according to the composition rule of fractional derivatives (Podlubny, 1998). Finally, we may obtain the scalar viscoacoustic wave equation with fractional derivatives.

We only present the asymptotic point-source radiation for the scalar viscoacoustic wave equation. For the vector wave equations 18, 19 and 20, the unknown quantities are the particle displacement, the normal stresses, and the momentum density (or the normal stresses), respectively. We may also adopt the asymptotic approximation to derive the point radiations for these vector wave equations. In this case, we need to formulate the Christoffel style equations to determine the polarization vectors for the unknown quantities. These polarization vectors correspond to the plane wave solution of these vector wave equations without source. The exact point-source solution is expressed as a superposition of all plane waves with different amplitudes, polarizations, phases and propagation directions. We may next utilize the asymptotic approximation shown in this paper to derive the point-source radiation for the vector wave equations. Similar treatment for viscoelastic anisotropic media can be found in Vavryčuk (2007a) and Shekar and Tsvankin (2014). 


\section{CONCLUSIONS}

The viscoacoustic wave equations in the scalar and vector forms are derived under the acoustic approximation (i.e. the shear-wave frequency-dependent velocity parameter $v_{S 0}$ equals zero) for a viscoacoustic orthorhombic medium. All of these viscoacoustic wave equations do not rely on a specified viscoacoustic model and can reduce to the ones for a nonattenuating orthorhombic medium. For the Kelvin-Voigt, Maxwell, SLS and Kjartansson models, the differential form of these vector wave equations can be obtained by introducing auxiliary variables.

As an approximation, the acoustic asymptotic radiation from a point source describes the acoustic wavefield at a far propagation distance. The result from the point-source asymptotic radiation demonstrates that attenuation due to energy absorption is an unignorable factor decaying wave amplitudes in attenuating media. For a frequency-dependent viscoacoustic model, the late-arrival trough of a waveform excited by a Ricker-wavelet point source is decayed more significantly than the early-arrival trough. The effect of the attenuationanisotropy parameters on the acoustic waveforms generally increases with the deviation of the propagation direction from the vertical axis. The attenuation-anisotropy parameters do not affect the arrival times of the waves in a viscoacoustic orthorhombic medium.

We will take into account the numerical implementation of the viscoacoustic wave equations in future papers.

\section{ACKNOWLEDGMENTS}

We are grateful to the assistant editor John Etgen, the anonymous associate editor and four anonymous reviewers for their reviews and comments. Most of the research work was 
accomplished when the first author was employed as a research consultant at KAUST. We thank KAUST for support. The first author also thanks the support from the College of Petroleum Engineering and Geosciences at KFUPM.

\section{APPENDIX A - PARAMETERIZATION OF AN ACOUSTIC ATTENUATING ORTHORHOMBIC MEDIUM}

As illustrated in the main text, we use the modified Alkhalifah's (2003) and Zhu and Tsvankin's (2007) notations to describe an acoustic attenuating orthorhombic medium. The symmetry planes of the medium are orthogonal to the coordinate axes. Let $\rho$ denote the density, $c_{i j}=c_{i j}^{R}-i \operatorname{sgn}(\omega) c_{i j}^{I}$ denote the complex-valued stiffness coefficients, and

$Q_{i j}=c_{i j}^{R} / c_{i j}^{I}$ denote the elements of the quality factor matrix. Note that the minus sign in front of the " $i$ " is opposite to the definition of the stiffness coefficients by (Zhu and Tsvankin, 2007) due to different conventions for the Fourier transform over $t$. The parameterization of an acoustic attenuating orthorhombic medium is shown as follows.

- $v_{P 0}$ : the velocity of the vertically propagating $\mathrm{P}$-wave

$$
v_{P 0} \equiv \sqrt{\frac{c_{33}^{R}}{\rho}} .
$$

- $v_{n 1}$ and $\eta_{1}$ : the NMO-velocity and anellipticity defined in the $[y, z]$ symmetry plane (where the subscript " 1 " denotes the $x$-axis normal to the plane)

$$
\begin{gathered}
v_{n 1} \equiv v_{P 0} \sqrt{1+2 \delta_{1}}=\sqrt{\frac{\left(c_{23}^{R}\right)^{2}+2 c_{23}^{R} c_{44}^{R}+c_{33}^{R} c_{44}^{R}}{\rho\left(c_{33}^{R}-c_{44}^{R}\right)}}, \\
\eta_{1} \equiv \frac{\epsilon_{1}-\delta_{1}}{1+2 \delta_{1}}=\frac{c_{22}^{R}\left(c_{33}^{R}-c_{44}^{R}\right)}{2\left[\left(c_{23}^{R}\right)^{2}+2 c_{23}^{R} c_{44}^{R}+c_{33}^{R} c_{44}^{R}\right]}-\frac{1}{2},
\end{gathered}
$$


where $\epsilon_{1}$ and $\delta_{1}$ denote the Thomsen-style velocity-anisotropy parameters defined in the $[y, z]$ plane (Tsvankin, 1997).

- $v_{n 2}$ and $\eta_{2}$ : the NMO-velocity and anellipticity defined in the $[x, z]$ symmetry plane (where the subscript " 2 " denotes the $y$-axis normal to the plane)

$$
\begin{gathered}
v_{n 2} \equiv v_{P 0} \sqrt{1+2 \delta_{2}}=\sqrt{\frac{\left(c_{13}^{R}\right)^{2}+2 c_{13}^{R} c_{55}^{R}+c_{33}^{R} c_{55}^{R}}{\rho\left(c_{33}^{R}-c_{55}^{R}\right)}}, \\
\eta_{2} \equiv \frac{\epsilon_{2}-\delta_{2}}{1+2 \delta_{2}}=\frac{c_{11}^{R}\left(c_{33}^{R}-c_{55}^{R}\right)}{2\left[\left(c_{13}^{R}\right)^{2}+2 c_{13}^{R} c_{55}^{R}+c_{33}^{R} c_{55}^{R}\right]}-\frac{1}{2},
\end{gathered}
$$

where $\epsilon_{2}$ and $\delta_{2}$ denote the Thomsen-style velocity-anisotropy parameters defined in the $[x, z]$ plane (Tsvankin, 1997).

- $\eta_{3}$ : the anellipticity defined in the $[x, y]$ plane (where the subscript " 3 " denotes the $z$-axis normal to the plane)

$$
\eta_{3} \equiv \frac{\epsilon_{1}-\epsilon_{2}-\delta_{3}\left(1+2 \epsilon_{2}\right)}{\left(1+2 \epsilon_{2}\right)\left(1+2 \delta_{3}\right)}=\frac{c_{22}^{R}\left(c_{11}^{R}-c_{66}^{R}\right)}{2\left[\left(c_{12}^{R}\right)^{2}+2 c_{12}^{R} c_{66}^{R}+c_{11}^{R} c_{66}^{R}\right]}-\frac{1}{2},
$$

where $\delta_{3}$ denotes the Thomsen-style velocity-anisotropy parameters defined in the $[x, y]$ plane (Tsvankin, 1997). The definition of $\eta_{3}$ in equation A-6 follows Grechka and Tsvankin (1999).

- $A_{P 0}$ : the wavenumber-normalized attenuation coefficient of the vertically propagating P-wave

$$
A_{P 0} \equiv Q_{33}\left(\sqrt{1+\frac{1}{Q_{33}^{2}}}-1\right)
$$

- $\epsilon_{Q 1}$ and $\delta_{Q 1}$ : the Thomsen-style parameters for attenuation anisotropy defined in the $[y, z]$ symmetry plane 


$$
\begin{gathered}
\epsilon_{Q 1} \equiv \frac{Q_{33}-Q_{22}}{Q_{22}}, \\
\delta_{Q 1} \equiv \frac{\frac{Q_{33}-Q_{44}}{Q_{44}} c_{44}^{R} \frac{\left(c_{23}^{R}+c_{33}^{R}\right)^{2}}{c_{33}^{R}-c_{44}^{R}}+2 \frac{Q_{33}-Q_{23}}{Q_{23}} c_{23}^{R}\left(c_{23}^{R}+c_{44}^{R}\right)}{c_{33}^{R}\left(c_{33}^{R}-c_{44}^{R}\right)},
\end{gathered}
$$

- $\epsilon_{Q 2}$ and $\delta_{Q 2}$ : the Thomsen-style parameters for attenuation anisotropy defined in the $[x, z]$ symmetry plane

$$
\begin{gathered}
\epsilon_{Q 2} \equiv \frac{Q_{33}-Q_{11}}{Q_{11}}, \\
\delta_{Q 2} \equiv \frac{\frac{Q_{33}-Q_{55}}{Q_{55}} c_{55}^{R} \frac{\left(c_{13}^{R}+c_{33}^{R}\right)^{2}}{c_{33}^{R}-c_{55}^{R}}+2 \frac{Q_{33}-Q_{13}}{Q_{13}} c_{13}^{R}\left(c_{13}^{R}+c_{55}^{R}\right)}{c_{33}^{R}\left(c_{33}^{R}-c_{55}^{R}\right)},
\end{gathered}
$$

- $\delta_{Q 3}$ : the Thomsen-style parameter for attenuation anisotropy defined in the $[x, y]$ symmetry plane

$$
\delta_{Q 3} \equiv \frac{\frac{Q_{11}-Q_{66}}{Q_{66}} c_{66}^{R} \frac{\left(c_{11}^{R}+c_{12}^{R}\right)^{2}}{c_{11}^{R}-c_{66}^{R}}+2 \frac{Q_{11}-Q_{12}}{Q_{12}} c_{12}^{R}\left(c_{12}^{R}+c_{66}^{R}\right)}{c_{11}^{R}\left(c_{11}^{R}-c_{66}^{R}\right)} .
$$

All of the above attenuation-anisotropy parameters are not applicable to the limit case $Q_{i j}=\infty$

\section{APPENDIX B - DERIVATION OF THE VECTOR ACOUSTIC WAVE EQUATIONS}

In this appendix, we show the derivation of the vector acoustic wave equations 18 and 19. As

mentioned in the section "Viscoacoustic orthorhombic models and the corresponding wave equations", we take into account the acoustic approximation $\left(v_{S 0}=0\right)$ for the stiffness coefficients in all frequencies. As a result, the stiffness coefficients $c_{44}(\omega), c_{55}(\omega)$ and $c_{66}(\omega)$ are always zero, which leads to the corresponding relaxation functions $\psi_{44}(t)=\psi_{55}(t)=$ $\psi_{66}(t)=0$. 
The relation between the strain $e_{i j}$ and the particle displacement $u_{i}$ is given by

$$
e_{x x}=\frac{\partial u_{x}}{\partial x}, \quad e_{y y}=\frac{\partial u_{y}}{\partial y}, \quad e_{z z}=\frac{\partial u_{z}}{\partial z}
$$

The constitutive equation is written as

$$
\begin{aligned}
& \sigma_{x x}=\psi_{11} \odot e_{x x}+\psi_{12} \odot e_{y y}+\psi_{13} \odot e_{z z}, \\
& \sigma_{y y}=\psi_{12} \odot e_{x x}+\psi_{22} \odot e_{y y}+\psi_{23} \odot e_{z z}, \\
& \sigma_{z z}=\psi_{13} \odot e_{x x}+\psi_{23} \odot e_{y y}+\psi_{33} \odot e_{z z},
\end{aligned}
$$

where $\sigma_{i j}$ and $\psi_{i j}$ denote the stress components and the relaxation functions, respectively.

The equation of motion is

$$
\begin{aligned}
& \rho \frac{\partial^{2} u_{x}}{\partial t^{2}}=\frac{\partial \sigma_{x x}}{\partial x}, \\
& \rho \frac{\partial^{2} u_{y}}{\partial t^{2}}=\frac{\partial \sigma_{y y}}{\partial y}, \\
& \rho \frac{\partial^{2} u_{z}}{\partial t^{2}}=\frac{\partial \sigma_{z z}}{\partial z},
\end{aligned}
$$

where $\rho$ denotes the density.

Substituting equations B-2 and B-1 successively into equation B-3 leads to the viscoacoustic wave equation in terms of the displacements,

$$
\begin{aligned}
\frac{\partial^{2} u_{x}}{\partial t^{2}} & =\phi_{11} \odot \frac{\partial^{2} u_{x}}{\partial x^{2}}+\phi_{12} \odot \frac{\partial^{2} u_{y}}{\partial x \partial y}+\phi_{13} \odot \frac{\partial^{2} u_{z}}{\partial x \partial z}, \\
\frac{\partial^{2} u_{y}}{\partial t^{2}} & =\phi_{12} \odot \frac{\partial^{2} u_{x}}{\partial x \partial y}+\phi_{22} \odot \frac{\partial^{2} u_{y}}{\partial y^{2}}+\phi_{23} \odot \frac{\partial^{2} u_{z}}{\partial y \partial z}, \\
\frac{\partial^{2} u_{z}}{\partial t^{2}} & =\phi_{13} \odot \frac{\partial^{2} u_{x}}{\partial x \partial z}+\phi_{23} \odot \frac{\partial^{2} u_{y}}{\partial y \partial z}+\phi_{33} \odot \frac{\partial^{2} u_{z}}{\partial z^{2}},
\end{aligned}
$$

where $\phi_{i j}=\psi_{i j} / \rho$ denote the density-normalized relaxation functions.

Taking the second-order derivative of equation B-2 with respect to the time and substituting equations B-1 and B-3, we derive the viscoacoustic wave equation in terms of the 
normal stresses,

$$
\begin{aligned}
& \frac{\partial^{2} \sigma_{x x}}{\partial t^{2}}=\phi_{11} \odot \frac{\partial^{2} \sigma_{x x}}{\partial x^{2}}+\phi_{12} \odot \frac{\partial^{2} \sigma_{y y}}{\partial y^{2}}+\phi_{13} \odot \frac{\partial^{2} \sigma_{z z}}{\partial z^{2}}, \\
& \frac{\partial^{2} \sigma_{y y}}{\partial t^{2}}=\phi_{12} \odot \frac{\partial^{2} \sigma_{x x}}{\partial x^{2}}+\phi_{22} \odot \frac{\partial^{2} \sigma_{y y}}{\partial y^{2}}+\phi_{23} \odot \frac{\partial^{2} \sigma_{z z}}{\partial z^{2}}, \\
& \frac{\partial^{2} \sigma_{z z}}{\partial t^{2}}=\phi_{13} \odot \frac{\partial^{2} \sigma_{x x}}{\partial x^{2}}+\phi_{23} \odot \frac{\partial^{2} \sigma_{y y}}{\partial y^{2}}+\phi_{33} \odot \frac{\partial^{2} \sigma_{z z}}{\partial z^{2}} .
\end{aligned}
$$

Here, we have assumed $e_{i j}(\mathbf{x}, 0)=0$ and $\dot{e}_{i j}(\mathbf{x}, 0)=0$ to use the following property,

$$
\frac{\partial^{2}}{\partial t^{2}}\left(\phi_{i j} \odot e_{j j}\right)=\phi_{i j} \odot \frac{\partial^{2} e_{j j}}{\partial t^{2}}
$$

where the dot on $\dot{e}_{i j}$ denotes the first temporal derivative, and the repeated indexes do not satisfy the Einstein summation convention.

The derivation of the above property is shown in Appendix C.

\section{APPENDIX C - DERIVATION OF EQUATION B-6}

This appendix derives equation B-6 in the case that the density-normalized relaxation functions are bounded when the time approaches zero from the positive time axis, that is $\left|\phi_{i j}(0+)\right|<\infty$. In the case of $\left|\phi_{i j}(0+)\right|=\infty$ for the Kjartansson model, we only need to disregard the term including $\phi_{i j}(0+)$ in the Riemann-Stieltjes convolution integral by referring to equation 8 , and the derivation can be accomplished in a similar way.

By change of variables in integral, equation 7 is rewritten as

$$
\psi_{i j k l}(t) \odot e_{k l}(\mathbf{x}, t)=\psi_{i j k l}(0+) e_{k l}(\mathbf{x}, t)+\int_{0}^{t} \dot{\psi}_{i j k l}(\tau) e_{k l}(\mathbf{x}, t-\tau) \mathrm{d} \tau .
$$

Taking account of equation $\mathrm{C}-1$, the first derivative of $\phi_{i j} \odot e_{j j}$ with respect to $t$ is written as

$$
\begin{aligned}
\frac{\partial}{\partial t}\left(\phi_{i j} \odot e_{j j}\right) & =\phi_{i j}(0+) \dot{e}_{j j}(\mathbf{x}, t)+\frac{\partial}{\partial t} \int_{0}^{t} \dot{\phi}_{i j}(\tau) e_{j j}(\mathbf{x}, t-\tau) d \tau \\
& =\phi_{i j}(0+) \dot{e}_{j j}(\mathbf{x}, t)+\dot{\phi}_{i j}(t) e_{j j}(\mathbf{x}, 0)+\int_{0}^{t} \dot{\phi}_{i j}(\tau) \dot{e}_{j j}(\mathbf{x}, t-\tau) d \tau
\end{aligned}
$$


where the dot on $\dot{e}_{j j}$ denotes the first derivative of $e_{j j}$ with respect to $t$.

Since no initial strain exists in the medium, we may assume $e_{i j}(\mathbf{x}, 0)=0$ so that

$$
\frac{\partial}{\partial t}\left(\phi_{i j} \odot e_{j j}\right)=\phi_{i j}(0+) \dot{e}_{j j}(\mathbf{x}, t)+\int_{0}^{t} \dot{\phi}_{i j}(\tau) \dot{e}_{j j}(\mathbf{x}, t-\tau) d \tau
$$

Taking the first derivative of equation C-3 with respect to $t$ and further assuming $\dot{e}_{j j}(\mathbf{x}, 0)=0$, we derive the following property

$$
\frac{\partial^{2}}{\partial t^{2}}\left(\phi_{i j} \odot e_{j j}\right)=\phi_{i j}(0+) \ddot{e}_{j j}(\mathbf{x}, t)+\int_{0}^{t} \dot{\phi}_{i j}(\tau) \ddot{e}_{j j}(\mathbf{x}, t-\tau) d \tau
$$

where the double dots on $\ddot{e}_{j j}$ denotes the second-order derivative of $e_{j j}$ with respect to $t$.

Referring to equation $\mathrm{C}-1$, the property can be written in a compact form as

$$
\frac{\partial^{2}}{\partial t^{2}}\left(\phi_{i j} \odot e_{j j}\right)=\phi_{i j} \odot \frac{\partial^{2} e_{j j}}{\partial t^{2}}
$$

\section{APPENDIX D - VISCOACOUSTIC WAVE EQUATIONS FOR TRANSVERSE ISOTROPY}

For a viscoacoustic VTI medium, we utilize the following medium parameters at the reference frequency: $\bar{v}_{P 0}, \bar{v}_{n}, \bar{\eta}, \bar{A}_{P 0}, \bar{\epsilon}_{Q}$ and $\bar{\delta}_{Q}$. The meaning of these parameters can be known by analogy with that of the similar parameters defined in the $[x, z]$ plane of an attenuating orthorhombic medium in Appendix A.

Taking into account the relation between acoustic VTI and orthorhombic anisotropies, we simplify equation 11 and obtain the nonzero density-normalized VTI stiffness parameters:

$$
\begin{aligned}
& a_{11}=a_{12}=a_{22}=v_{n}^{2}\left[1-2 i k_{Q}\left(1+\epsilon_{Q}\right)\right](1+2 \eta), \\
& a_{13}=a_{23}=v_{P 0} v_{n}\left(1-2 i k_{Q}\right)-i k_{Q} \delta_{Q} \frac{v_{P 0}^{3}}{v_{n}} \\
& a_{33}=v_{P 0}^{2}\left(1-2 i k_{Q}\right)
\end{aligned}
$$


where $k_{Q}$ is given by equation 12 .

Using these density-normalized stiffness coefficients, we may obtain the density-normalized VTI relaxation functions for the viscoacoustic models shown in the section "viscoacoustic orthorhombic models and the corresponding wave equations". The density-normalized relaxation functions for a viscoacoustic VTI medium satisfy

$$
\phi_{11}(t)=\phi_{12}(t)=\phi_{22}(t), \quad \phi_{13}(t)=\phi_{23}(t) .
$$

Utilizing equation D-1, we simplify the orthorhombic dispersion relation (equation 14) and finally obtain the VTI dispersion relation (Hao and Alkhalifah, 2017b). Implementing the inverse Fourier transform to the VTI dispersion relation, we derive the scalar viscoacoustic VTI wave equation:

$$
\begin{aligned}
\frac{\partial^{4} P}{\partial t^{4}} & =\phi_{11} \odot\left(\frac{\partial^{4} P}{\partial t^{2} \partial x^{2}}+\frac{\partial^{4} P}{\partial t^{2} \partial y^{2}}\right)+\phi_{33} \odot \frac{\partial^{4} P}{\partial t^{2} \partial z^{2}} \\
& +\left(\phi_{13} \odot \phi_{13}-\phi_{11} \odot \phi_{33}\right) \odot\left(\frac{\partial^{4} P}{\partial x^{2} \partial z^{2}}+\frac{\partial^{2} P}{\partial y^{2} \partial z^{2}}\right) .
\end{aligned}
$$

Taking equation D-2 into account, we may simplify the vector viscoacoustic orthorhombic wave equation 18-20. Finally, the vector viscoacoustic VTI wave equation are as follows.

The wave equations in terms of the particle displacement are given by

$$
\begin{aligned}
& \frac{\partial^{2} u_{x}}{\partial t^{2}}=\phi_{11} \odot \frac{\partial^{2} u_{x}}{\partial x^{2}}+\phi_{11} \odot \frac{\partial^{2} u_{y}}{\partial x \partial y}+\phi_{13} \odot \frac{\partial^{2} u_{z}}{\partial x \partial z}, \\
& \frac{\partial^{2} u_{y}}{\partial t^{2}}=\phi_{11} \odot \frac{\partial^{2} u_{x}}{\partial x \partial y}+\phi_{11} \odot \frac{\partial^{2} u_{y}}{\partial y^{2}}+\phi_{13} \odot \frac{\partial^{2} u_{z}}{\partial y \partial z}, \\
& \frac{\partial^{2} u_{z}}{\partial t^{2}}=\phi_{13} \odot \frac{\partial^{2} u_{x}}{\partial x \partial z}+\phi_{13} \odot \frac{\partial^{2} u_{y}}{\partial y \partial z}+\phi_{33} \odot \frac{\partial^{2} u_{z}}{\partial z^{2}},
\end{aligned}
$$

where $\mathbf{u}=\left(u_{x}, u_{y}, u_{z}\right)^{T}$ denotes the particle displacement.

The wave equations in terms of the normal stresses are given by

$$
\begin{aligned}
\frac{\partial^{2} \sigma_{h h}}{\partial t^{2}} & =\phi_{11} \odot\left(\frac{\partial^{2} \sigma_{h h}}{\partial x^{2}}+\frac{\partial^{2} \sigma_{h h}}{\partial y^{2}}\right)+\phi_{13} \odot \frac{\partial^{2} \sigma_{z z}}{\partial z^{2}}, \\
\frac{\partial^{2} \sigma_{z z}}{\partial t^{2}} & =\phi_{13} \odot\left(\frac{\partial^{2} \sigma_{h h}}{\partial x^{2}}+\frac{\partial^{2} \sigma_{h h}}{\partial y^{2}}\right)+\phi_{33} \odot \frac{\partial^{2} \sigma_{z z}}{\partial z^{2}},
\end{aligned}
$$


where $\sigma_{h h}=\sigma_{x x}=\sigma_{y y}$ denotes the horizontal normal stress.

The wave equations in terms of the momentum density and the normal stresses are given by

$$
\begin{gathered}
\frac{\partial J_{x}}{\partial t}=\frac{\partial \sigma_{h h}}{\partial x}, \\
\frac{\partial J_{y}}{\partial t}=\frac{\partial \sigma_{h h}}{\partial y}, \\
\frac{\partial J_{z}}{\partial t}=\frac{\partial \sigma_{z z}}{\partial z}, \\
\frac{\partial \sigma_{h h}}{\partial t}=\phi_{11} \odot \frac{\partial J_{x}}{\partial x}+\phi_{11} \odot \frac{\partial J_{y}}{\partial y}+\phi_{13} \odot \frac{\partial J_{z}}{\partial z}, \\
\frac{\partial \sigma_{z z}}{\partial t}=\phi_{13} \odot \frac{\partial J_{x}}{\partial x}+\phi_{13} \odot \frac{\partial J_{y}}{\partial y}+\phi_{33} \odot \frac{\partial J_{z}}{\partial z}
\end{gathered}
$$

where $\mathbf{J}=\left(J_{x}, J_{y}, J_{z}\right)^{T}$ is the momentum density $(\mathbf{J}=\rho \partial \mathbf{u} / \partial t)$ according to Auld (1973). 


\section{REFERENCES}

Alkhalifah, T., 2000, An acoustic wave equation for anisotropic media: Geophysics, 65, $1239-1250$.

- , 2003, An acoustic wave equation for orthorhombic anisotropy: Geophysics, 68, 11691172.

Apostol, T. M., 1974, Mathematical analysis (2nd ed.): Addison-Wesley.

Arfken, G. B., and H. J. Weber, 2001, Mathematical methods for physicists (5th ed.): Academic Press.

Auld, B. A., 1973, Acoustic fields and waves in solids: Wiley-Interscience publication.

Bai, T., and I. Tsvankin, 2016, Time-domain finite-difference modeling for attenuative anisotropic media: Geophysics, 81, C69-C77.

Bai, T., I. Tsvankin, and X. Wu, 2017, Waveform inversion for attenuation estimation in anisotropic media: Geophysics, 82, WA83-WA93.

Ben-Menahem, A., and S. J. Singh, 1981, Seismic waves and sources: Springer-Verlag.

Bleistein, N., 2012, Saddle point contribution for an n-fold complex-valued integral: Center for Wave Phenomena Research Report, 741-750.

Borcherdt, R. D., 2009, Viscoelastic waves in layered media: Cambridge University Press.

Buchwald, V. T., 1959, Elastic waves in anisotropic media: Proceedings of the Royal Society. Series A, Mathematical, Physical and Engineering Sciences, 253, 563-580.

Burridge, R., P. Chadwick, and A. N. Norris, 1993, Fundamental elastodynamic solutions for anisotropic media with ellipsoidal slowness surfaces: Proceedings of the Royal Society. Series A, Mathematical, Physical and Engineering Sciences, 440, 655-681.

Carcione, J. M., 2015, Wave fields in real media: Theory and numerical simulation of wave propagation in anisotropic, anelastic, porous and electromagnetic media: Handbook of 
geophysical exploration (3rd ed.): Elsevier.

Carcione, J. M., F. Cavallini, F. Mainardi, and A. Hanyga, 2002, Time-domain modeling of constant-Q seismic waves using fractional derivatives: Pure and Applied Geophysics, 159, 1719-1736.

Christensen, R., 1982, Theory of viscoelasticity: an introduction (2nd ed.): Elsevier.

da Silva, N. V., G. Yao, and M. Warner, 2019, Wave modeling in viscoacoustic media with transverse isotropy: Geophysics, 84, C41-C56.

Duveneck, E., and P. M. Bakker, 2011, Stable P-wave modeling for reverse-time migration in tilted TI media: Geophysics, 76, S65-S75.

Fowler, P., and R. King, 2011, Modeling and reverse time migration of orthorhombic pseudoacoustic P-waves: 81st Annual International Meeting, SEG, Expanded Abstracts, 190195.

Grechka, V., and I. Tsvankin, 1999, 3-D moveout velocity analysis and parameter estimation for orthorhombic media: Geophysics, 64, 820-837.

Gurtin, M. E., and E. Sternberg, 1962, On the linear theory of viscoelasticity: Archive for Rational Mechanics and Analysis, 11, 291-356.

Hao, Q., and T. Alkhalifah, 2017a, An acoustic eikonal equation for attenuating orthorhombic media: Geophysics, 82, WA67-WA81.

,$- 2017 \mathrm{~b}$, An acoustic eikonal equation for attenuating transversely isotropic media with a vertical symmetry axis: Geophysics, 82, C9-C20.

Hudson, J. A., 1980, The excitation and propagation of elastic waves: Cambridge University Press.

Kjartansson, 1979, Constant Q-wave propagation and attenuation: Journal of Geophysical Research, 84, 4737-4748. 
Lakes, R., 2009, Viscoelastic materials: Cambridge University Press.

Lighthill, M. J., 1960, Studies on magneto-hydrodynamic waves and other anisotropic wave motions: Philosophical Transitions of the Royal Society. Series A, Mathematical, Physical and Engineering Sciences, 252, 397-430.

Mainardi, F., 2010, Fractional calculus and waves in linear viscoelasticity: an introduction to mathematical models: Imperial College Press.

Pipkin, A. C., 1986, Lectures on viscoelasticity theory (2nd ed.): Springer-Verlag.

Podlubny, I., 1998, Fractional differential equations: an introduction to fractional derivatives, fractional differential equations, to methods of their solution and some of their applications: Elsevier.

Qu, Y., J. Huang, Z. Li, Z. Guan, and J. Li, 2017, Attenuation compensation in anisotropic least-squares reverse time migration: Geophysics, 82, S411-S423.

Schiessel, H., R. Metzler, A. Blumen, and T. Nonnenmacher, 1995, Generalized viscoelastic models: their fractional equations with solutions: Journal of Physics A: Mathematical and General, 28, 6567 .

Shekar, B., and I. Tsvankin, 2014, Point-source radiation in attenuative anisotropic media: Geophysics, 79, WB25-WB34.

Stovas, A., 2015, Azimuthally dependent kinematic properties of orthorhombic media: Geophysics, 80, C107-C122.

Tschoegl, N. W., 1989, The phenomenological theory of linear viscoelastic behavior: An introduction: Springer-Verlag.

Tsvankin, I., 1997, Anisotropic parameters and P-wave velocity for orthorhombic media: Geophysics, 62, 1292-1309.

van der Hijden, J. H. M. T., 1987, Radiation from an impulsive line source in an unbounded 
homogeneous anisotropic medium: Geophysical Journal of the Royal Astronomical Society, 91, 355-372.

Vavryčuk, V., 1997, Elastodynamic and elastostatic green tensors for homogeneous weak transversely isotropic media: Geophysical Journal International, 130, 786-800.

— , 2007a, Asymptotic Green's function in homogeneous anisotropic viscoelastic media: Proceedings of the Royal Society A: Mathematical, Physical and Engineering Sciences, 463, 2689-2707.

— $2007 \mathrm{~b}$, Ray velocity and ray attenuation in homogeneous anisotropic viscoelastic media: Geophysics, 72, D119-D127.

Wang, C.-Y., and J. D. Archenbach, 1995, Three-dimensional time-harmonic elastodynamic green's functions for anisotropic solids: Proceedings of the Royal Society of London. Series A, Mathematical and Physical Sciences, 449, 441-458.

Xu, W.-C., G.-Q. Yang, Z.-C. Li, X.-D. Sun, and J. Wang, 2016, Pseudo acoustic equation for TI medium attenuation based on the GSLS model: Chinese Journal of Geophysics, 59, 2232-2244.

Yeatts, F. R., 1984, Elastic radiation from a point source in an anisotropic medium: Physical Review Letters B, 29, 1674-1684.

Zhu, T., 2017, Numerical simulation of seismic wave propagation in viscoelastic-anisotropic media using frequency-independent Q wave equation: Geophysics, 82, WA1-WA10.

Zhu, T., and T. Bai, 2019, Efficient modeling of wave propagation in a vertical transversely isotropic attenuative medium based on fractional laplacian: Geophysics, 84, T121-T131.

Zhu, Y., and I. Tsvankin, 2006, Plane-wave propagation in attenuative transversely isotropic media: Geophysics, 71, T17-T30.

— 2007, Plane-wave attenuation anisotropy in orthorhombic media: Geophysics, 72, 
D9-D19. 


\section{LIST OF TABLES}

1 The medium parameters at the reference frequency $f_{c}=40 \mathrm{~Hz}$, where the value

of $\bar{A}_{P 0}$ corresponds to $\bar{Q}_{33}=30.0$. The bar on the medium parameters corresponds to the reference frequency. 


\section{LIST OF FIGURES}

1 A causal signal $S(t)$. The signal $S(t)$ is the Ricker wavelet with the dominant frequency of $40 \mathrm{~Hz}$. The fourth-order derivative of $S(t)$ is used as the source wavelet in the section "Numerical examples".

2 The acoustic asymptotic radiation from a point source in the viscoacoustic orthorhombic models. All of the waveforms are observed at the propagation distance $r=1 \mathrm{~km}$. $\alpha$ and $\beta$ denote the polar and azimuthal angles of the observation direction, measured from the $z$ - and $x$-axes, respectively. The red, blue, orange, green, dashed black lines correspond to the Kelvin-Voigt, Maxwell, SLS, Kjartansson and frequency-independent viscoacoustic orthorhombic models. For the Kelvin-Voigt, Maxwell, SLS and Kjartansson models, the medium parameters at the reference frequency are shown at Table 1 . The medium parameters for the frequency-independent viscoacoustic model are the same as the parameters shown at Table 1 . The signal $S(t)$ in Figure 1 is used to obtain the point source for all of these models.

3 A comparison between the point-source radiations from the SLS and Kjartansson models. The orange and green lines correspond to the SLS and Kjartansson models, respectively. These lines are the same as those in Figure 2.

4 A comparison of the point-source radiations from the nonattenuating orthorhombic model and the viscoacoustic orthorhombic models. All of the waveforms are observed at the propagation distance $r=1 \mathrm{~km} . \alpha$ and $\beta$ denote the polar and azimuthal angles of the observation direction. The parameters of the viscoacoustic orthorhombic models at the reference frequency are shown in Table 1 . The cyan lines correspond to the nonattenuating model. The other colored lines are the same as those in Figure 2. The signal $S(t)$ in Figure 1 is used to obtain the point source for all these models. 
5 A comparison of the point-source radiations from two viscoacoustic models. One (corresponding to the orange lines) is the SLS model with anisotropic attenuation coefficients, and the model parameters are shown in Table 1. The other one (corresponding to the gray lines $)$ is the similar model but with isotropic attenuation coefficients $\left(\epsilon_{Q 1}=\delta_{Q 1}=\right.$ $\left.\epsilon_{Q 2}=\delta_{Q 2}=\delta_{Q 3}=0\right)$. All of the waveforms are observed at the propagation distance $r=1 \mathrm{~km} . \alpha$ and $\beta$ denote the polar and azimuthal angles of the observation direction. The signal $S(t)$ in Figure 1 is used to obtain the point source for both models. 


\begin{tabular}{cccccc}
\hline $\bar{v}_{P 0}(\mathrm{~km} / \mathrm{s})$ & $\bar{v}_{n 1}(\mathrm{~km} / \mathrm{s})$ & $\bar{v}_{n 2}(\mathrm{~km} / \mathrm{s})$ & $\bar{\eta}_{1}$ & $\bar{\eta}_{2}$ & $\bar{\eta}_{3}$ \\
\hline 3.0 & 2.846 & 3.286 & 0.278 & 0.167 & 0.229 \\
\hline $\bar{A}_{P 0}$ & $\bar{\epsilon}_{Q 1}$ & $\bar{\delta}_{Q 1}$ & $\bar{\epsilon}_{Q 2}$ & $\bar{\delta}_{Q 2}$ & $\bar{\delta}_{Q 3}$ \\
\hline 0.0167 & 0.66 & 0.52 & -0.33 & 0.98 & 0.94 \\
\hline
\end{tabular}

Table 1: The medium parameters at the reference frequency $f_{c}=40 \mathrm{~Hz}$, where the value of $\bar{A}_{P 0}$ corresponds to $\bar{Q}_{33}=30.0$. The bar on the medium parameters corresponds to the reference frequency. 


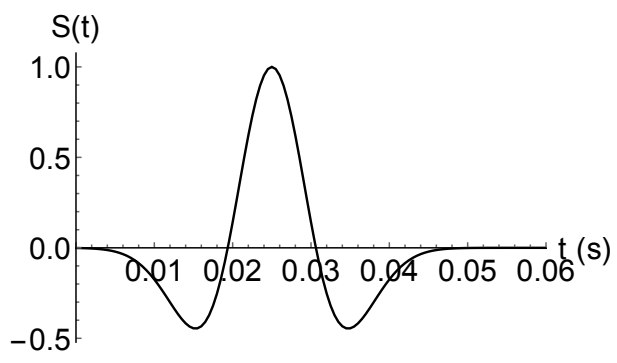

Figure 1: A causal signal $S(t)$. The signal $S(t)$ is the Ricker wavelet with the dominant frequency of $40 \mathrm{~Hz}$. The fourth-order derivative of $S(t)$ is used as the source wavelet in the section "Numerical examples". 


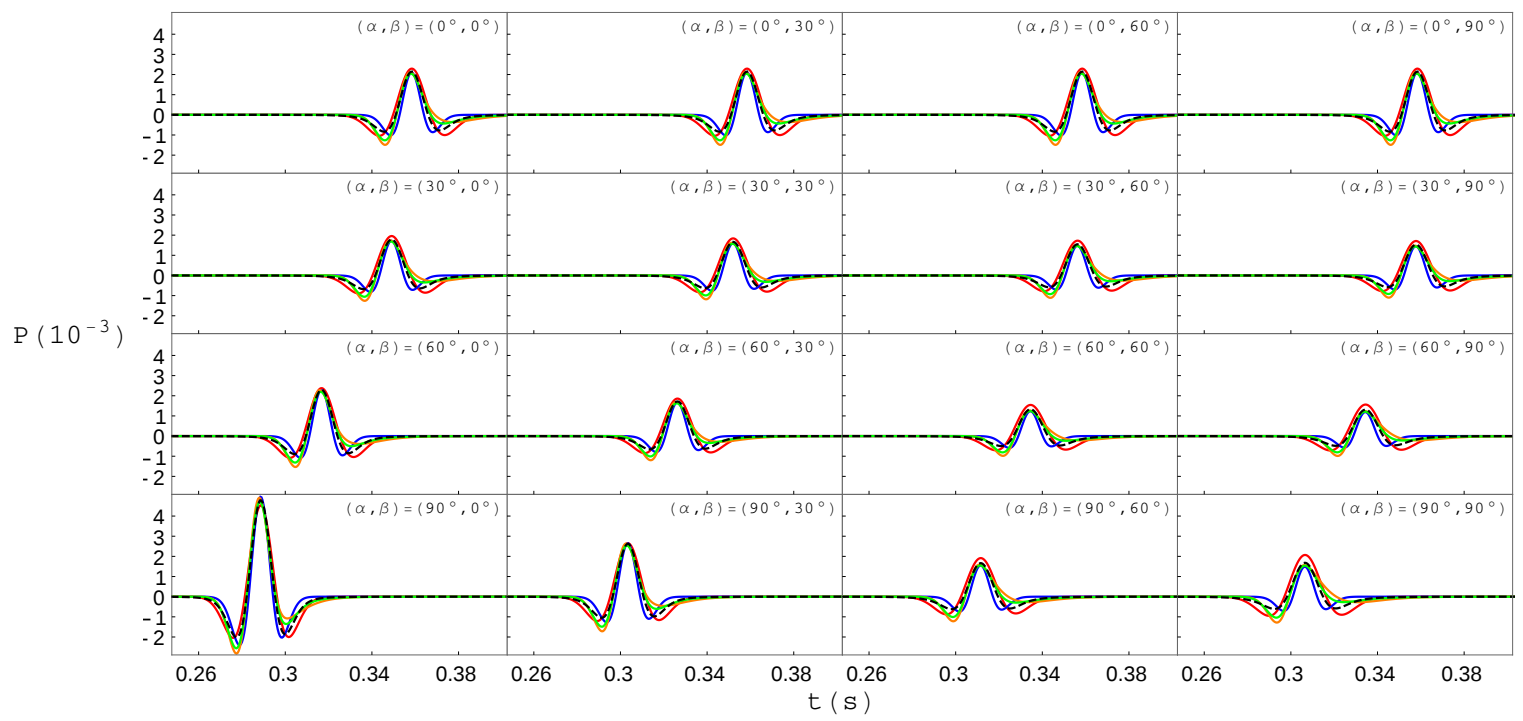

Figure 2: The acoustic asymptotic radiation from a point source in the viscoacoustic orthorhombic models. All of the waveforms are observed at the propagation distance $r=$ $1 \mathrm{~km} . \alpha$ and $\beta$ denote the polar and azimuthal angles of the observation direction, measured from the $z$ - and $x$-axes, respectively. The red, blue, orange, green, dashed black lines correspond to the Kelvin-Voigt, Maxwell, SLS, Kjartansson and frequency-independent viscoacoustic orthorhombic models. For the Kelvin-Voigt, Maxwell, SLS and Kjartansson models, the medium parameters at the reference frequency are shown at Table 1 . The medium parameters for the frequency-independent viscoacoustic model are the same as the parameters shown at Table 1 . The signal $S(t)$ in Figure 1 is used to obtain the point source for all of these models. 


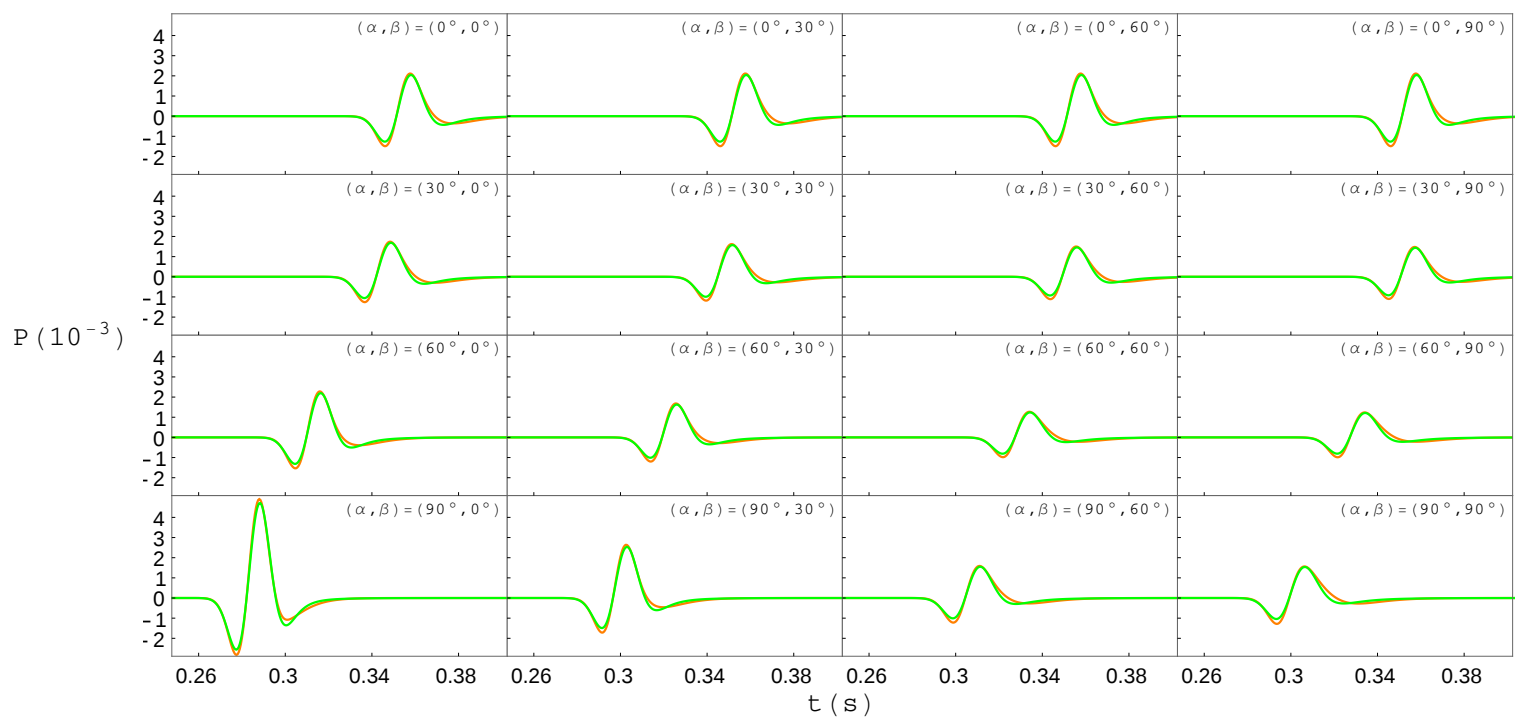

Figure 3: A comparison between the point-source radiations from the SLS and Kjartansson models. The orange and green lines correspond to the SLS and Kjartansson models, respectively. These lines are the same as those in Figure 2. 


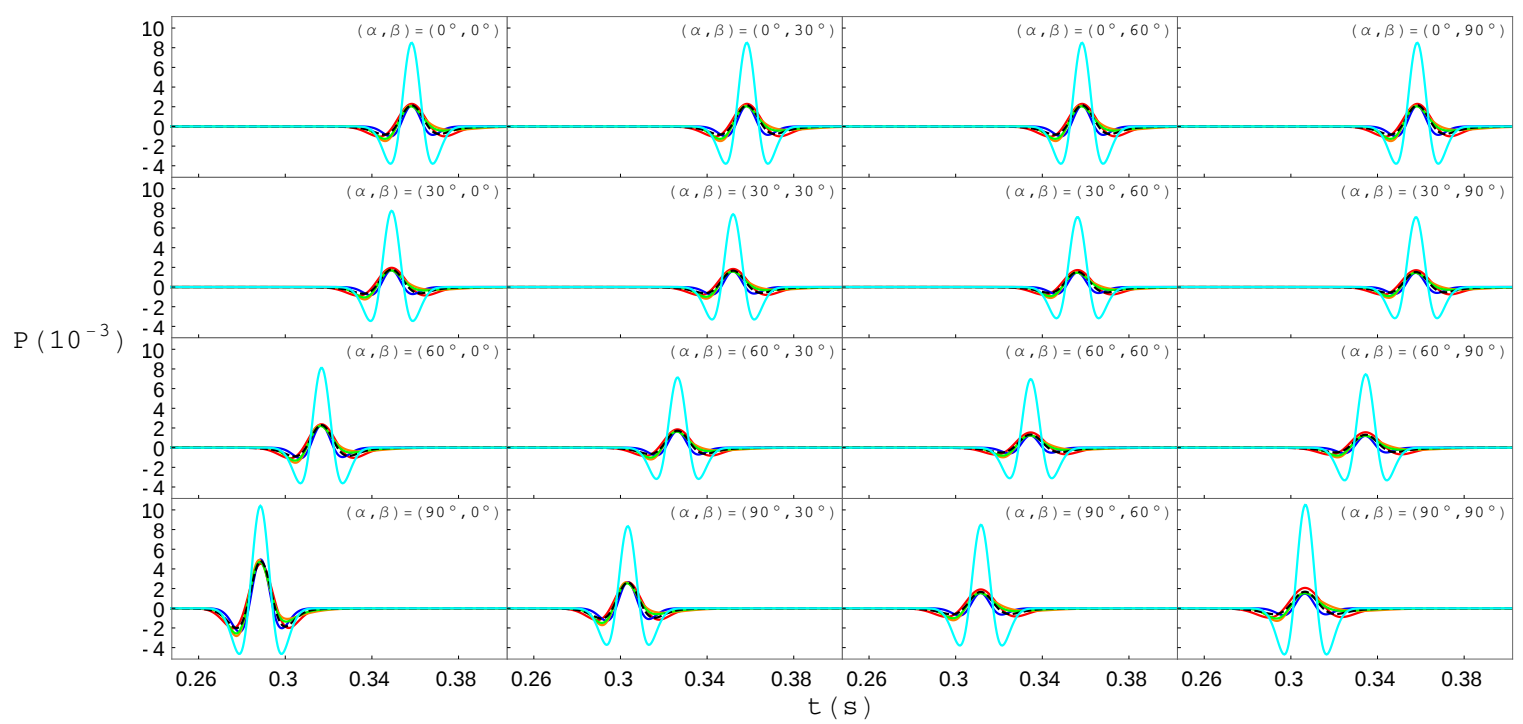

Figure 4: A comparison of the point-source radiations from the nonattenuating orthorhombic model and the viscoacoustic orthorhombic models. All of the waveforms are observed at the propagation distance $r=1 \mathrm{~km} . \alpha$ and $\beta$ denote the polar and azimuthal angles of the observation direction. The parameters of the viscoacoustic orthorhombic models at the reference frequency are shown in Table 1 . The cyan lines correspond to the nonattenuating model. The other colored lines are the same as those in Figure 2. The signal $S(t)$ in Figure 1 is used to obtain the point source for all these models. 


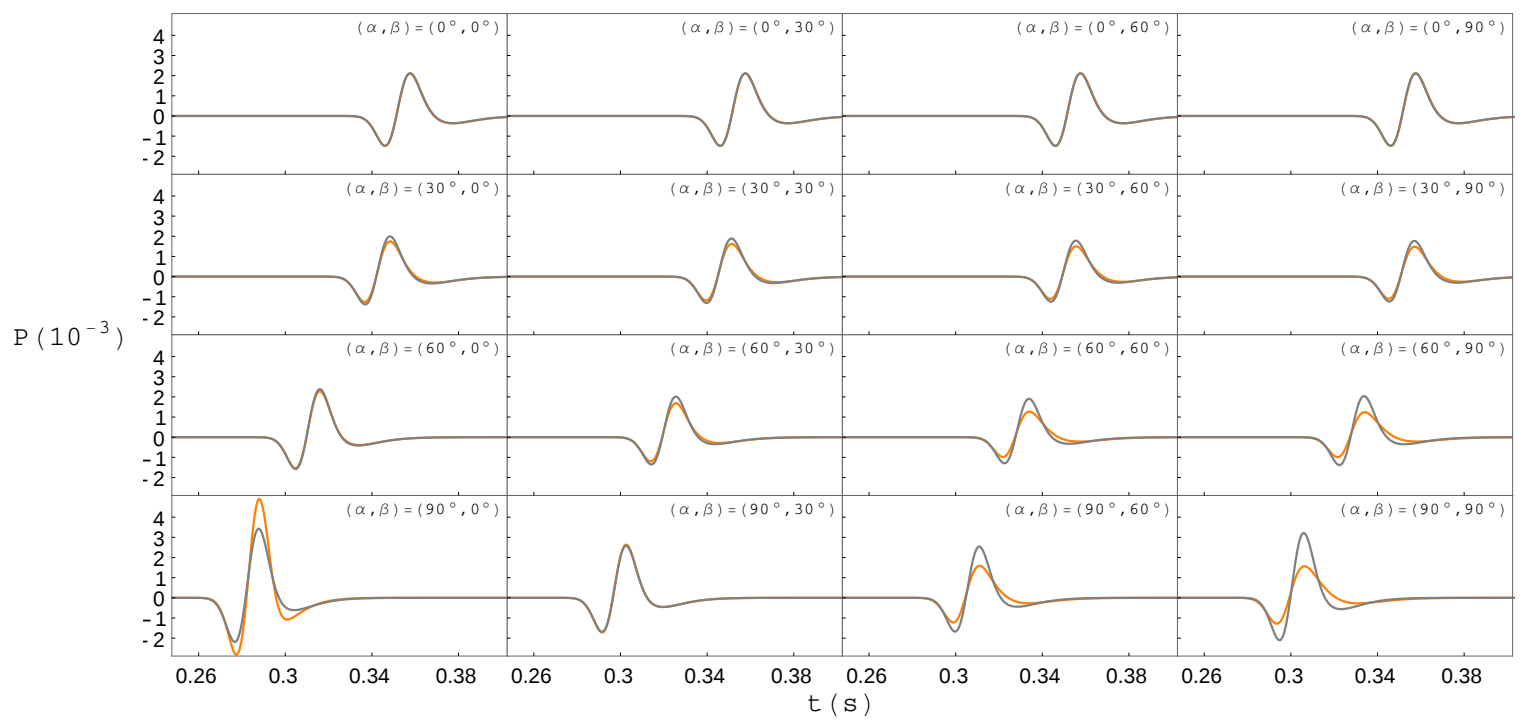

Figure 5: A comparison of the point-source radiations from two viscoacoustic models. One (corresponding to the orange lines) is the SLS model with anisotropic attenuation coefficients, and the model parameters are shown in Table 1. The other one (corresponding to the gray lines) is the similar model but with isotropic attenuation coefficients $\left(\epsilon_{Q 1}=\right.$ $\left.\delta_{Q 1}=\epsilon_{Q 2}=\delta_{Q 2}=\delta_{Q 3}=0\right)$. All of the waveforms are observed at the propagation distance $r=1 \mathrm{~km} . \alpha$ and $\beta$ denote the polar and azimuthal angles of the observation direction. The signal $S(t)$ in Figure 1 is used to obtain the point source for both models. 